\title{
The genus of a division algebra and the unramified Brauer group
}

\author{
Vladimir I. Chernousov • Andrei S. Rapinchuk • \\ Igor A. Rapinchuk
}

Received: 17 March 2013 / Accepted: 27 March 2013 / Published online: 27 April 2013 (C) The Author(s) 2013. This article is published with open access at SpringerLink.com

\begin{abstract}
Let $D$ be a finite-dimensional central division algebra over a field $K$. We define the genus gen $(D)$ of $D$ to be the collection of classes $\left[D^{\prime}\right] \in \operatorname{Br}(K)$, where $D^{\prime}$ is a central division $K$-algebra having the same maximal subfields as $D$. In this paper, we describe a general approach to proving the finiteness of $\operatorname{gen}(D)$ and estimating its size that involves the unramified Brauer group with respect to an appropriate set of discrete valuations of $K$. This approach is then implemented in some concrete situations, yielding in particular an extension of the Stability Theorem of A. Rapinchuk and I. Rapinchuk (Manuscr. Math. 132:273-293, 2010) from quaternion algebras to arbitrary algebras of exponent two. We also consider an example where the size of the genus can be estimated explicitly. Finally, we offer two generalizations of the genus problem for division algebras: one deals with absolutely almost simple algebraic $K$ groups having the same isomorphism/isogeny classes of maximal $K$-tori, and the other with the analysis of weakly commensurable Zariski-dense subgroups.
\end{abstract}

Keywords Division algebra $\cdot$ Maximal field · Brauer group ·

Linear algebraic group $\cdot$ Maximal torus

Communicated by Efim Zelmanov.

V. I. Chernousov

Department of Mathematical Sciences, University of Alberta, Edmonton, AB T6G 2G1, Canada

e-mail: vladimir@ualberta.ca

\section{A. S. Rapinchuk (凶)}

Department of Mathematics, University of Virginia, Charlottesville, VA 22904-4137, USA

e-mail: asr3x@virginia.edu

I. A. Rapinchuk

Department of Mathematics, Yale University, New Haven, CT 06520-8283, USA

e-mail: igor.rapinchuk@yale.edu 
Mathematics Subject Classification (2010) $\quad 20 \mathrm{G} 15 \cdot 20 \mathrm{G} 10 \cdot 12 \mathrm{E} 15 \cdot 11 \mathrm{R} 52$

\section{Introduction}

For a finite-dimensional central division algebra $A$ over a field $K$, we let $[A]$ denote the corresponding class in the Brauer group $\operatorname{Br}(K)$ of $K$. Following [5], we define the genus $\operatorname{gen}(D)$ of a central division $K$-algebra $D$ of degree $n$ to be the collection of all classes $\left[D^{\prime}\right] \in \operatorname{Br}(K)$, where $D^{\prime}$ is a central division $K$-algebra having the same maximal fields as $D$ (in precise terms, this means that $D^{\prime}$ has the same degree $n$, and a field extension $P / K$ of degree $n$ admits a $K$-embedding $P \hookrightarrow D$ if and only if it admits a $K$-embedding $P \hookrightarrow D^{\prime}$ ). One of the results announced in [5] states that if $K$ is a finitely generated field, then the genus $\operatorname{gen}(D)$ of a central division $K$-algebra $D$ of degree $n$ prime to char $K$ is finite. The proof consists of two parts: first, one relates the size of $\operatorname{gen}(D)$ to that of ${ }_{n} \operatorname{Br}(K)_{V}$, the $n$-torsion of the unramified Brauer group $\operatorname{Br}(K)_{V}$ with respect to a suitable set $V$ of discrete valuations of $K$; second, one establishes the finiteness of ${ }_{n} \operatorname{Br}(K)_{V}$. The goal of the current paper is to give a detailed exposition of the first part. This analysis, in particular, enables us to extend the Stability Theorem of [24] from quaternion algebras to arbitrary algebras of exponent two. In [5], we sketched a proof, communicated to us by J.-L. Colliot-Thélène [6], of the finiteness of ${ }_{n} \operatorname{Br}(K)_{V}$ for a suitable $V$, which relies on Deligne's finiteness theorem for constructible sheaves [7] and Gabber's purity theorem [8]. Our original proof was based on an analysis of the standard exact sequence for the Brauer group of a curve (cf. [16] or [9, 9.25, p. 27]), and the details of this proof will be given elsewhere. A noteworthy feature of the second proof is that it leads to explicit estimates on the order of the $n$-torsion of the unramified Brauer group, hence on the size of the genus: to demonstrate this point, as well as to showcase some of the ideas involved in the general argument, we compute an upper bound for the size of the 2-torsion in the unramified Brauer group of the field of rational functions of a split elliptic curve over a number field. In any case, the set of valuations $V$ for which one can prove the finiteness of ${ }_{n} \operatorname{Br}(K)_{V}$ is rather special and arises from geometric considerations; at the same time, one can relate the size of $\operatorname{gen}(D)$ to that of ${ }_{n} \operatorname{Br}(K)_{V}$ in a much more general context (which is our main motivation for separating the two parts of the argument). So, we begin with a precise description of the set-up that will be used throughout this paper.

Let $K$ be a field. Given a discrete valuation $v$ of $K$, we will denote by $\mathcal{O}_{K, v}$ and $\bar{K}_{v}$ its valuation ring and residue field, respectively. Fix an integer $n>1$ (which will later be either the degree or the exponent of $D$ ) and suppose that $V$ is a set of discrete valuations of $K$ that satisfies the following three conditions:

(A) For any $a \in K^{\times}$, the set $V(a):=\{v \in V \mid v(a) \neq 0\}$ is finite;

(B) There exists a finite subset $V^{\prime} \subset V$ such that the field of fractions of

$$
\mathcal{O}:=\bigcap_{v \in V \backslash V^{\prime}} \mathcal{O}_{K, v}
$$

coincides with $K$;

(C) For any $v \in V$, the characteristic of the residue field $\bar{K}_{v}$ is prime to $n$. 
(We note that if $K$ is finitely generated, which will be the case in most of our applications, then (B) automatically follows from (A)—see \$2). Due to (C), we can define for each $v \in V$ the corresponding residue map

$$
\rho_{v}:{ }_{n} \operatorname{Br}(K) \longrightarrow \operatorname{Hom}\left(\mathcal{G}^{(v)}, \mathbb{Z} / n \mathbb{Z}\right),
$$

where $\mathcal{G}^{(v)}$ is the absolute Galois group of $\bar{K}_{v}$ (cf., for example, [25, §10] or [26, Chap. 2, Appendix $]$ ). As usual, a class $[A] \in{ }_{n} \operatorname{Br}(K)$ (or a finite-dimensional central simple $K$-algebra $A$ representing this class) is said to be unramified at $v$ if $\rho_{v}([A])=1$, and ramified otherwise. We let $\operatorname{Ram}_{V}(A)$ (or $\operatorname{Ram}_{V}([A])$ ) denote the set of all $v \in V$ where $A$ is ramified; one shows that this set is always finite (Proposition 2.1). We also define the unramified part of ${ }_{n} \operatorname{Br}(K)$ with respect to $V$ to be

$$
{ }_{n} \operatorname{Br}(K)_{V}=\bigcap_{v \in V} \operatorname{Ker} \rho_{v} .
$$

The goal of $\$ 2$ is to prove the following result that relates the size of the genus with the order of the unramified Brauer group.

Theorem 2.2. Assume that ${ }_{n} \operatorname{Br}(K)_{V}$ is finite. Then for any finite-dimensional central division $K$-algebra $D$ of exponent $n$, the intersection $\operatorname{gen}(D) \cap{ }_{n} \operatorname{Br}(K)$ is finite, of size

$$
\left|\operatorname{gen}(D) \cap{ }_{n} \operatorname{Br}(K)\right| \leqslant\left|{ }_{n} \operatorname{Br}(K)_{V}\right| \cdot \varphi(n)^{r} \text {, with } r=\left|\operatorname{Ram}_{V}(D)\right| \text {, }
$$

where $\varphi$ is the Euler function. In particular, if $D$ has degree $n$ then

$$
|\operatorname{gen}(D)| \leqslant\left|{ }_{n} \operatorname{Br}(K)_{V}\right| \cdot \varphi(n)^{r}
$$

We use this result in $\S 3$ to estimate the size of the genus for division algebras over the function fields of curves in certain situations. This analysis, in particular, enables us to describe some cases where gen $(D)$ reduces to a single element. First, we observe that since the opposite algebra $D^{\text {op }}$ has the same maximal subfields as $D$, this can happen only if $\left[D^{\mathrm{op}}\right]=[D]$, i.e. if $D$ has exponent 2 in the Brauer group. On the other hand, it follows from the theorem of Artin-Hasse-Brauer-Noether (AHBN) (cf. Sect. 3.6) that gen $(D)$ does reduce to a single element for any algebra $D$ of exponent 2 over a global field $K$ (in which case $D$ is necessarily a quaternion algebra). The following theorem, which was established earlier in [24] for quaternion algebras, expands the class of fields with this property.

Theorem 3.5. (Stability Theorem) Let $k$ be a field of characteristic $\neq 2$.

(1) Suppose $k$ satisfies the following property:

(*) If $D$ and $D^{\prime}$ are central division $k$-algebras of exponent 2 having the same maximal subfields, then $D \simeq D^{\prime}$ (in other words, for any $D$ of exponent 2 , $\left.\left|\operatorname{gen}(D) \cap_{2} \operatorname{Br}(k)\right|=1\right)$.

Then the field of rational functions $k(x)$ also satisfies $(*)$. 
(2) If $|\operatorname{gen}(D)|=1$ for any central division $k$-algebra $D$ of exponent 2 , then the same is true for any central division $k(x)$-algebra of exponent 2.

Corollary 3.8. Let $k$ be either a finite field of characteristic $\neq 2$ or a number field, and $K=k\left(x_{1}, \ldots, x_{r}\right)$ be a finitely generated purely transcendental extension of $k$. Then for any central division $K$-algebra $D$ of exponent 2 , we have $|\operatorname{gen}(D)|=1$.

In $\S 4$, we will give explicit estimates on the size of the genus of a quaternion algebra over the field of rational functions of a split elliptic curve over a number field (cf. Theorem 4.1). Finally, in $\S \S 5,6$ we discuss possible generalizations of the finiteness theorem for the genus [5] in the context of general absolutely almost simple algebraic groups. More precisely, Conjecture 5.2 predicts the finiteness of the genus, defined in terms of the isomorphism classes of maximal $K$-tori, of an absolutely almost simple algebraic group over a finitely generated field $K$ of "good" characteristic-Theorem 5.3 confirms this conjecture for inner forms of type $A_{\ell}$. In $\S 6$, after a brief review of the notion of weak commensurability and its connections with length-commensurability of locally symmetric spaces (cf. [21], [23]), we formulate Conjecture 6.1 that asserts the finiteness of the number of forms of a given absolutely simple algebraic group over a finitely generated field $K$ of characteristic zero that can contain a finitely generated Zariski-dense subgroup with the trace field $K$ weakly commensurable to a given finitely generated Zariski-dense subgroup (both Conjecture 5.2 and 6.1 are true over number fields).

\section{Ramification places and the genus of a division algebra}

Let $K$ be a field. Fix an integer $n>1$ and let $V$ be a set of discrete valuations of $K$ satisfying conditions (A), (B) and (C) of $\S 1$ [we observe that if $K$ is generated over its prime subfield by nonzero elements $a_{1}, \ldots, a_{r}$, then using (A), one can find a finite subset $V^{\prime} \subset V$ such that $v\left(a_{i}\right)=0$ for all $v \in V \backslash V^{\prime}$ and all $i=1, \ldots, r$. Then $a_{1}, \ldots, a_{r}$ lie in $\mathcal{O}=\bigcap_{v \in V \backslash V^{\prime}} \mathcal{O}_{K, v}$, and hence the fraction field of the latter coincides with $K$. Thus, for a finitely generated field $K$, condition (B) follows automatically from condition $(\mathrm{A})]$.

Proposition 2.1 Assume that $V$ satisfies conditions (A), (B), and (C). Then for any $[A] \in{ }_{n} \operatorname{Br}(K)$, the set $\operatorname{Ram}_{V}([A])$ is finite.

Proof Pick a finite set $V^{\prime} \subset V$ as in (B), and set

$$
\mathcal{O}=\bigcap_{v \in V \backslash V^{\prime}} \mathcal{O}_{K, v} .
$$

It is enough to show that the set of $v \in V \backslash V^{\prime}$ where $A$ ramifies is finite. Let $\operatorname{dim}_{K} A=$ $\ell^{2}$. First, we note that it is possible to find a basis $x_{1}=1, \ldots, x_{\ell^{2}}$ of $A$ over $K$ such that

$$
\mathcal{A}:=\mathcal{O} x_{1}+\cdots+\mathcal{O} x_{\ell^{2}}
$$


is a subring of $A$. Indeed, let $y_{1}, \ldots, y_{\ell^{2}}$ be an arbitrary $K$-basis of $A$ with $y_{1}=1$. Then, we can write

$$
y_{i} y_{j}=\sum_{k=1}^{\ell^{2}} c_{i j}^{k} y_{k} \quad \text { with } c_{i j}^{k} \in K \text {. }
$$

Since $K$ is the field of fractions of $\mathcal{O}$, there exists $d \in \mathcal{O}$ such that $d c_{i j}^{k} \in \mathcal{O}$ for all $i, j$ and $k$. Multiplying (1) by $d^{2}$, we obtain

$$
\left(d y_{i}\right)\left(d y_{j}\right)=\sum_{k=1}^{\ell^{2}}\left(d c_{i j}^{k}\right)\left(d y_{k}\right),
$$

which implies that the basis $x_{1}=1, x_{2}=d y_{2}, \ldots, x_{\ell^{2}}=d y_{\ell^{2}}$ is as required.

Now, for $v \in V \backslash V^{\prime}$, we set

$$
A_{v}=A \otimes_{K} K_{v} \text { and } \mathcal{A}_{v}=\mathcal{A} \otimes_{\mathcal{O}} \mathcal{O}_{v}
$$

We have $A_{v}=\mathcal{A}_{v} \otimes \mathcal{O}_{v} K_{v}$, and furthermore if $\mathcal{A}_{v}$ is an Azumaya algebra, then $A$ is unramified at $v$ (cf. $[25, \S 10]$ ). Since $A$ is a central simple algebra over $K$, the canonical map $\varphi: A \otimes_{K} A^{\mathrm{op}} \rightarrow \operatorname{End}_{K} A$ given by

$$
\sum_{i=1}^{r} a_{i} \otimes b_{i} \longrightarrow\left(x \mapsto \sum_{i=1}^{r} a_{i} x b_{i}\right)
$$

is an isomorphism. Identifying $\mathcal{A} \otimes \mathcal{O} \mathcal{A}^{\mathrm{op}}$ and $\operatorname{End}_{\mathcal{O}} \mathcal{A}$ with $\mathcal{O}$-submodules of $A \otimes_{K} A^{\text {op }}$ and $\operatorname{End}_{K} A$, respectively, we observe that the fact that $\varphi$ is an isomorphism, in conjunction with the finite generation of the $\mathcal{O}$-module $\operatorname{End}_{\mathcal{O}} \mathcal{A} \simeq M_{\ell^{2}}(\mathcal{O})$, implies the existence of a nonzero $t \in \mathcal{O}$ such that

$$
t \cdot \operatorname{End}_{\mathcal{O}} \mathcal{A} \subset \varphi\left(\mathcal{A} \otimes_{\mathcal{O}} \mathcal{A}^{\mathrm{op}}\right)
$$

Now, suppose $v \in V$ lies outside of the finite set $V^{\prime} \cup V(t)$, and let $\varphi_{v}$ be the map analogous to $\varphi$ for the algebra $A_{v}$. Since $\operatorname{End}_{\mathcal{O}_{v}} \mathcal{A}_{v}=\left(\operatorname{End}_{\mathcal{O}} \mathcal{A}\right) \otimes_{\mathcal{O}} \mathcal{O}_{v}$ and $t \in \mathcal{O}_{v}^{\times}$, we conclude from (2) that

$$
\varphi_{v}\left(\mathcal{A}_{v} \otimes_{\mathcal{O}_{v}} \mathcal{A}_{v}^{\mathrm{op}}\right)=\operatorname{End}_{\mathcal{O}_{v}} \mathcal{A}_{v}
$$

This means that $\mathcal{A}_{v}$ is an Azumaya $\mathcal{O}_{v}$-algebra, from which our claim follows.

The main result of this section is the following.

Theorem 2.2 Assume that ${ }_{n} \operatorname{Br}(K)_{V}$ is finite. Then for any finite-dimensional central division K-algebra $D$ of exponent $n$, the intersection $\operatorname{gen}(D) \cap{ }_{n} \operatorname{Br}(K)$ is finite, of size 


$$
\left|\operatorname{gen}(D) \cap{ }_{n} \operatorname{Br}(K)\right| \leqslant\left|{ }_{n} \operatorname{Br}(K)_{V}\right| \cdot \varphi(n)^{r}, \text { with } r=\left|\operatorname{Ram}_{V}(D)\right| \text {, }
$$

where $\varphi$ is the Euler function. In particular, if $D$ has degree $n$ then

$$
|\operatorname{gen}(D)| \leqslant\left|{ }_{n} \operatorname{Br}(K)_{V}\right| \cdot \varphi(n)^{r}
$$

We begin the proof with the following generalization of Lemma 2.5 of [24], which establishes the desired conclusion without the assumption that the residue field satisfies condition (LD) introduced in loc. cit. We recall that given a finite-dimensional central division algebra $\mathcal{D}$ over a field $\mathcal{K}$ which is complete with respect to a discrete valuation $v$, the valuation $v$ uniquely extends to a discrete valuation $\tilde{v}$ of $\mathcal{D}$ (cf. [27, Chap. 12, $\S 2]$, [33]). Furthermore, the corresponding valuation ring $\mathcal{O}_{\mathcal{D}}$ has a unique maximal 2-sided ideal $\mathfrak{P}_{\mathcal{D}}$ (the valuation ideal), and the quotient $\overline{\mathcal{D}}=\mathcal{O}_{\mathcal{D}} / \mathfrak{P}_{\mathcal{D}}$ is a finitedimensional division (but not necessarily central) algebra, called the residue algebra, over the residue field $\overline{\mathcal{K}}$.

Lemma 2.3 Let $\mathcal{K}$ be a field complete with respect to a discrete valuation $v$ with residue field $k$. Suppose $\mathcal{D}_{1}$ and $\mathcal{D}_{2}$ are two finite-dimensional central division $\mathcal{K}$-algebras of degree $n$ prime to chark, and, for $i=1,2$, let $\mathcal{E}_{i}$ be the center of the residue algebra $\overline{\mathcal{D}}_{i}$. If $\mathcal{D}_{1}$ and $\mathcal{D}_{2}$ have the same maximal subfields, then $\mathcal{E}_{1}=\mathcal{E}_{2}$.

Proof Recall that $\mathcal{E}_{1}$ and $\mathcal{E}_{2}$ are cyclic Galois extensions of $k$ (cf. [34, Proposition 2.5]). By symmetry, to prove that $\mathcal{E}_{1}=\mathcal{E}_{2}$, it suffices to prove the existence of a $k$-embedding $\mathcal{E}_{1} \hookrightarrow \mathcal{E}_{2}$. Assume that there is no such embedding, and let $\mathcal{L}_{i}$ denote the unramified extension of $\mathcal{K}$ with residue field $\mathcal{E}_{i}$. Then $\mathcal{L}_{i}$ is a cyclic Galois extension of $\mathcal{K}$, and $\mathcal{L}_{1} \hookrightarrow \mathcal{L}_{2}$. By construction, $\mathcal{L}_{2}$ embeds in $\mathcal{D}_{2}$ and, since $\mathcal{D}_{1}$ and $\mathcal{D}_{2}$ have the same maximal subfields, $\mathcal{L}_{2}$ embeds in $\mathcal{D}_{1}$ as well. Let $\mathcal{L}_{2}^{(i)}$ be the image of some $\mathcal{K}$-embedding $\mathcal{L}_{2} \hookrightarrow \mathcal{D}_{i}$, and let $\Delta_{i}$ be the centralizer of $\mathcal{L}_{2}^{(i)}$ in $\mathcal{D}_{i}$. It is well-known (and follows from the proof of the Double Centralizer Theorem) that $\Delta_{i}$ is Brauerequivalent to $\mathcal{D}_{i} \otimes \mathcal{K} \mathcal{L}_{2}^{(i)}$. We now observe that the assumption that $\mathcal{L}_{1} \hookrightarrow \mathcal{L}_{2}$ implies that the $\mathcal{L}_{2}$-algebra $\mathcal{D}_{1} \otimes \mathcal{K} \mathcal{L}_{2}^{(1)}$ is ramified with respect to the extension $w$ of $v$ to $\mathcal{L}_{2}$. To see this, we will use the following well-known statement.

Theorem 2.4 ([25, Theorem 10.4]) Let $\mathcal{K}$ be a field complete with respect to a discrete valuation $v$, with residue field $k$, and let $n>1$ be an integer prime to char $k$. For $a$ finite extension $\mathcal{L} / \mathcal{K}$, we let $w$ and $\ell$ denote the extension of $v$ and the corresponding residue field. Then the following diagram

$$
\begin{gathered}
{ }_{n} \operatorname{Br}(\mathcal{L}) \stackrel{\rho_{w}}{\longrightarrow} \operatorname{Hom}\left(\mathcal{G}^{(w)}, \mathbb{Z} / n \mathbb{Z}\right) \\
\uparrow \\
\uparrow[e] \\
{ }_{n} \operatorname{Br}(\mathcal{K}) \stackrel{\rho_{v}}{\longrightarrow} \operatorname{Hom}\left(\mathcal{G}^{(v)}, \mathbb{Z} / n \mathbb{Z}\right)
\end{gathered}
$$

in which $\mathcal{G}^{(v)}$ and $\mathcal{G}^{(w)}$ are the absolute Galois groups of $k$ and $\ell$, respectively, $\rho_{v}$ and $\rho_{w}$ the corresponding residue maps, and $[e]$ denotes the composition of the natural restriction map with multiplication by the ramification index $e=e(w \mid v)$, commutes. 
It is well-known that the subgroup of the absolute Galois group $\mathcal{G}^{(v)}$ of $k$ fixing $\mathcal{E}_{1}$ coincides with $\operatorname{Ker} \rho_{v}\left(\left[\mathcal{D}_{1}\right]\right)\left(\left[34\right.\right.$, Theorem 3.5]). So, the assumption that $\mathcal{E}_{1} \hookrightarrow \mathcal{E}_{2}$ means that the restriction of $\rho_{v}\left(\left[\mathcal{D}_{1}\right]\right)$ to the subgroup $\mathcal{G}^{(w)}$ of $\mathcal{G}^{(v)}$ corresponding to $\mathcal{E}_{2}$, is nontrivial. Since $e(w \mid v)=1$, Theorem 2.4 implies that $\rho_{w}\left(\left[\mathcal{D}_{1} \otimes_{\mathcal{K}} \mathcal{L}_{2}\right]\right)$ is nontrivial, i.e. $\mathcal{D}_{1} \otimes \mathcal{K} \mathcal{L}_{2}^{(1)}$ is ramified at $w$, as claimed.

Let $\tilde{w}$ be the extension of $w$ to $\Delta_{1}$. Since $\left[\Delta_{1}\right]=\left[\mathcal{D}_{1} \otimes \mathcal{K} \mathcal{L}_{2}^{(1)}\right]$ is ramified at $w$, the ramification index $e(\tilde{w} \mid w)$ is $>1$ (cf. [34, Theorem 3.4]; note that being of degree prime to chark, the division algebra $\Delta_{1}$ is "inertially split"). It follows that $\Delta_{1}$ contains a maximal subfield $\mathcal{P}$ which is ramified over $\mathcal{L}_{2}^{(1)}$. By our assumption, $\mathcal{P}$ embeds into $\mathcal{D}_{2}$, and moreover, by the Skolem-Noether theorem, we may assume that this embedding maps $\mathcal{L}_{2}^{(1)}$ onto $\mathcal{L}_{2}^{(2)}$, hence its image (which we will also denote by $\mathcal{P}$ ) is contained in $\Delta_{2}$. Since $\mathcal{P} / \mathcal{L}_{2}^{(2)}$ is ramified, we conclude that the $\mathcal{L}_{2}$-algebra $\Delta_{2}$, and hence also $\mathcal{D}_{2} \otimes \mathcal{K} \mathcal{L}_{2}$, is ramified with respect to $w$. On the other hand, it follows from Theorem 2.4 that $\mathcal{D}_{2} \otimes \mathcal{K} \mathcal{L}_{2}$ is unramified at $w$, a contradiction.

Lemma 2.5 Let $D$ and $D^{\prime}$ be central division $K$-algebras such that $[D] \in{ }_{n} \operatorname{Br}(K)$ and $\left[D^{\prime}\right] \in \operatorname{gen}(D) \cap{ }_{n} \operatorname{Br}(K)$. Given $v \in V$, we let $\chi_{v}$ and $\chi_{v}^{\prime} \in \operatorname{Hom}\left(\mathcal{G}^{(v)}, \mathbb{Z} / n \mathbb{Z}\right)$ denote the images of $[D]$ and $\left[D^{\prime}\right]$, respectively, under the residue map $\rho_{v}$. Then

$$
\operatorname{Ker} \chi_{v}=\operatorname{Ker} \chi_{v}^{\prime}
$$

for all $v \in V$.

Proof Write

$$
D \otimes_{K} K_{v}=M_{\ell}(\mathcal{D}) \text { and } D^{\prime} \otimes_{K} K_{v}=M_{\ell^{\prime}}\left(\mathcal{D}^{\prime}\right),
$$

where $\mathcal{D}$ and $\mathcal{D}^{\prime}$ are central division algebras over $\mathcal{K}=K_{v}$. According to [24, Corollary 2.4], we have $\ell=\ell^{\prime}$ and $\mathcal{D}$ and $\mathcal{D}^{\prime}$ have the same maximal subfields. Letting $\mathcal{E}$ and $\mathcal{E}^{\prime}$ denote the centers of the residue algebras $\overline{\mathcal{D}}$ and $\overline{\mathcal{D}}^{\prime}$, respectively, we infer from Lemma 2.3 that $\mathcal{E}=\mathcal{E}^{\prime}$ (note that the lemma applies since by assumption $n$ is relatively prime to char $\bar{K}_{v}$ ). On the other hand, as we already mentioned in the proof of Lemma $2.3, \operatorname{Ker} \chi_{v}$ and $\operatorname{Ker} \chi_{v}^{\prime}$ are precisely the subgroups of $\mathcal{G}^{(v)}$ corresponding to $\mathcal{E}$ and $\mathcal{E}^{\prime}$, respectively (cf. [34, Theorem 3.5]). So, our claim follows.

Proof of Theorem 2.2 Suppose that $\left[D^{\prime}\right] \in \operatorname{gen}(D) \cap{ }_{n} \operatorname{Br}(K)$. Fix $v \in V$, and set $\chi_{v}=\rho_{v}([D])$ and $\chi_{v}^{\prime}=\rho_{v}\left(\left[D^{\prime}\right]\right)$. According to Lemma 2.5, we have

$$
\operatorname{Ker} \chi_{v}=\operatorname{Ker} \chi_{v}^{\prime}
$$

Let $m$ be the order of $\chi_{v}$. Any character $\chi_{v}^{\prime}$ of $\mathcal{G}^{(v)}$ satisfying (3) can be viewed as a faithful character of the order $m$ cyclic group $\mathcal{G}^{(v)} / \operatorname{Ker} \chi_{v}$, and therefore there are $\varphi(m)$ possibilities for $\chi^{\prime}$. So,

$$
\left|\rho_{v}\left(\operatorname{gen}(D) \cap{ }_{n} \operatorname{Br}(K)\right)\right| \leqslant \varphi(m) \leqslant \varphi(n)
$$

for any $v \in V$ (as $m$ divides $n)$, and 


$$
\rho_{v}\left(\operatorname{gen}(D) \cap{ }_{n} \operatorname{Br}(K)\right)=\{1\}
$$

if $\rho_{v}([D])=1$.

Now, since, by Proposition 2.1, any division algebra ramifies at a finite number of places, we can consider the map

$$
\rho_{V}:{ }_{n} \operatorname{Br}(K) \longrightarrow \bigoplus_{v \in V} \operatorname{Hom}\left(\mathcal{G}^{(v)}, \mathbb{Z} / n \mathbb{Z}\right), \rho=\left(\rho_{v}\right)
$$

Our previous discussion shows that

$$
\left|\rho_{V}\left(\operatorname{gen}(D) \cap_{n} \operatorname{Br}(K)\right)\right| \leqslant \varphi(n)^{r}
$$

where $r=\left|\operatorname{Ram}_{V}(D)\right|$. By definition $\operatorname{Ker} \rho_{V}={ }_{n} \operatorname{Br}(K)_{V}$, so we obtain the required estimate.

Now, if $D$ has degree $n$, then clearly $\operatorname{gen}(D) \subset{ }_{n} \operatorname{Br}(K)$, and our second assertion follows from the first one.

Remark 2.6 1. Our proof of Theorem 2.2 actually leads to the following somewhat stronger assertion, which will be used in $\S 5$. Let $K$ be a field, and $V$ be a set of discrete valuations of $K$ satisfying conditions (A), (B) and (C) for a given integer $n>1$. For a central division algebra $D$ of degree $n$ over $K$, we define the local

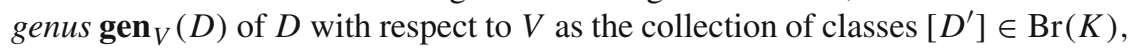
where $D^{\prime}$ is a central division algebra $K$-algebra of degree $n$ such that for any $v \in V$, if one writes $D \otimes_{K} K_{v}=M_{\ell}(\mathcal{D})$ and $D^{\prime} \otimes_{K} K_{v}=M_{\ell^{\prime}}\left(\mathcal{D}^{\prime}\right)$ where $\mathcal{D}$ and $\mathcal{D}^{\prime}$ are central division algebras over $K_{v}$, then $\ell=\ell^{\prime}$ and $\mathcal{D}$ and $\mathcal{D}^{\prime}$ have the same maximal separable subfields. If $n$ is prime to char $K$ and ${ }_{n} \operatorname{Br}(K)_{V}$ is finite, then $\operatorname{gen}_{V}(D)$ is finite for any central division $K$-algebra $D$ of degree $n$.

2. Lemma 2.3 remains valid (and so do its consequences) if one replaces the assumption that the degree $n$ is prime to char $k$ by the weaker assumption that $\mathcal{E}_{1}$ and $\mathcal{E}_{2}$ are separable extensions of $k$. This makes $\mathcal{D}_{1}$ and $\mathcal{D}_{2}$ "inertially split," and the argument goes through without any significant changes.

\section{The genus over the function fields of curves}

We will now apply the results of $\S 2$ in the case where $K=k(C)$ is the field of rational functions on a smooth absolutely irreducible projective curve $C$ over a field $k$, and $V$ is the set of all geometric places of $K$, i.e. those discrete valuations of $K$ that are trivial on $k$. If $n>1$ is an integer prime to chark, then it is clear that $V$ satisfies conditions (A), (B) and (C). The corresponding unramified Brauer ${ }_{n} \operatorname{Br}(K)_{V}$ will then, following tradition, be denoted by ${ }_{n} \operatorname{Br}(K)_{\text {ur }}$ (it is known that this is precisely the $n$-torsion subgroup of the Brauer group of the curve $C$, cf. [16]). Note that there is a natural map $\iota_{k}:{ }_{n} \operatorname{Br}(k) \rightarrow{ }_{n} \operatorname{Br}(K)$ ur. The following theorem provides an estimation of the size of $\operatorname{gen}(D) \cap{ }_{n} \operatorname{Br}(K)$ for a central division $K$-algebra $D$ of exponent $n$ in certain situations. 
Theorem 3.1 Let $n>1$ be an integer prime to chark. Assume that

- the set $C(k)$ of rational points is infinite;

- $\left.\mid{ }_{n} \operatorname{Br}(K)\right)_{\mathrm{ur}} / \iota_{k}\left({ }_{n} \operatorname{Br}(k)\right) \mid=: M<\infty$.

Then

(1) if there exists $N<\infty$ such that

$$
\left|\operatorname{gen}(\Delta) \cap{ }_{n} \operatorname{Br}(k)\right| \leqslant N
$$

for any central division $k$-algebra $\Delta$ of exponent dividing $n$, then for any central division $K$-algebra $D$ of exponent $n$ we have

$$
\left|\operatorname{gen}(D) \cap{ }_{n} \operatorname{Br}(K)\right| \leqslant M \cdot N \cdot \varphi(n)^{r},
$$

where $r=\left|\operatorname{Ram}_{V}(D)\right|$;

(2) if $\operatorname{gen}(\Delta) \cap{ }_{n} \operatorname{Br}(k)$ is finite for any central division $k$-algebra $\Delta$ of exponent dividing $n$, then $\operatorname{gen}(D) \cap{ }_{n} \operatorname{Br}(K)$ is finite for any central division $K$-algebra $D$ of exponent $n$.

Proof Let $D$ be a finite-dimensional central division $K$-algebra such that $[D] \in$ ${ }_{n} \operatorname{Br}(K)$, and set $r=\left|\operatorname{Ram}_{V}(D)\right|$. Arguing as in the proof of Theorem 2.2, we see that in the notations introduced therein, we have

$$
\left|\rho_{V}\left(\operatorname{gen}(D) \cap{ }_{n} \operatorname{Br}(K)\right)\right| \leqslant \varphi(n)^{r},
$$

i.e., $\operatorname{gen}(D) \cap{ }_{n} \operatorname{Br}(K)$ is contained in a union of $\leqslant \varphi(n)^{r}$ cosets modulo ${ }_{n} \operatorname{Br}(K)_{\text {ur }}$. It follows that $\operatorname{gen}(D) \cap_{n} \operatorname{Br}(K)$ is contained in a union of $\leqslant M \cdot \varphi(n)^{r}$ cosets modulo $\iota_{k}\left({ }_{n} \operatorname{Br}(k)\right)$. Thus, it is enough to show that for $\left[D^{\prime}\right] \in \operatorname{gen}(D)$ of exponent $n$, the intersection $\operatorname{gen}(D) \cap\left(\left[D^{\prime}\right] \cdot \iota_{k}\left({ }_{n} \operatorname{Br}(k)\right)\right)$ is

- finite if $\operatorname{gen}(\Delta) \cap_{n} \operatorname{Br}(k)$ is finite for any central division $k$-algebra $\Delta$ with $[\Delta] \in$ ${ }_{n} \operatorname{Br}(k)$;

- of size $\leqslant N$ if $N<\infty$ has the property that $\left|\operatorname{gen}(\Delta) \cap_{n} \operatorname{Br}(k)\right| \leqslant N$ for any $\Delta$ as above.

Notice that for $\left[D^{\prime}\right] \in \operatorname{gen}(D)$, we have $\operatorname{gen}\left(D^{\prime}\right)=\operatorname{gen}(D)$, so, to simplify our notations, we may replace $D^{\prime}$ by $D$. Then, our problem reduces to proving the above two statements for the intersection

$$
\Lambda:=\operatorname{gen}(D) \cap\left([D] \cdot \iota_{k}\left({ }_{n} \operatorname{Br}(k)\right)\right),
$$

where $D$ is any finite-dimensional central division $K$-algebra such that $[D] \in{ }_{n} \operatorname{Br}(K)$.

For this, we first recall (cf., for example, [27, Chap. 12, §3] or [34, §3]) that given a field $\mathcal{K}$ complete with respect to a discrete valuation $v$ and an integer $n>1$ prime to the characteristic of the residue field $\overline{\mathcal{K}}$, there is a natural isomorphism $v$ between the unramified Brauer group ${ }_{n} \operatorname{Br}(\mathcal{K})_{\{v\}}$ and ${ }_{n} \operatorname{Br}(\overline{\mathcal{K}})$. This isomorphism can be described 
as follows: if $[\mathcal{D}] \in{ }_{n} \operatorname{Br}(\mathcal{K})_{\{v\}}$ is represented by a central division $\mathcal{K}$-algebra $\mathcal{D}$, then the residue division algebra $\overline{\mathcal{D}}$ is central over $\overline{\mathcal{K}}$ and $v([\mathcal{D}])=[\overline{\mathcal{D}}]$.

Now let $D$ be a finite-dimensional central division algebra over $K$ such that $[D] \in$ ${ }_{n} \operatorname{Br}(K)$. Since $C(k)$ is infinite, we can pick $v \in V$ such that $\bar{K}_{v}=k$ and $D$ is unramified at $v$. Let $\mathcal{K}=K_{v}$ be the completion of $K$ with respect to $v$, and define $v_{v}:{ }_{n} \operatorname{Br}(K)\{v\} \rightarrow{ }_{n} \operatorname{Br}(k)$ to be the composition of the natural map ${ }_{n} \operatorname{Br}(K)_{\{v\}} \rightarrow$ ${ }_{n} \operatorname{Br}(\mathcal{K})_{\{v\}}$ with the isomorphism $v:{ }_{n} \operatorname{Br}(\mathcal{K})_{\{v\}} \rightarrow{ }_{n} \operatorname{Br}(k)$ constructed above (note that $\overline{\mathcal{K}}=k)$. Pick any $\left[D^{\prime}\right] \in \operatorname{gen}(D) \cap{ }_{n} \operatorname{Br}(K)$, and write

$$
D \otimes_{K} \mathcal{K}=M_{\ell}(\mathcal{D}) \text { and } D^{\prime} \otimes_{K} \mathcal{K}=M_{\ell^{\prime}}\left(\mathcal{D}^{\prime}\right),
$$

with $\mathcal{D}$ and $\mathcal{D}^{\prime}$ division $\mathcal{K}$-algebras. As in the proof of Lemma 2.5, we infer from [24, Corollary 2.4] that $\ell=\ell^{\prime}$ and $\mathcal{D}$ and $\mathcal{D}^{\prime}$ have the same maximal subfields. Furthermore, it follows from Lemma 2.5 that $D^{\prime}$ (equivalently, $\mathcal{D}^{\prime}$ ) is unramified at $v$. Let $\Delta=\overline{\mathcal{D}}$ and $\Delta^{\prime}=\overline{\mathcal{D}}^{\prime}$ be the corresponding residue algebras (which are central division algebras over $k$ of the same dimension $\left.d^{2}=\operatorname{dim}_{\mathcal{K}} \mathcal{D}=\operatorname{dim}_{\mathcal{K}} \mathcal{D}^{\prime}\right)$. We claim that $\Delta$ and $\Delta^{\prime}$ have the same maximal subfields. Indeed, let $\mathcal{O}_{\mathcal{D}}$ and $\mathcal{O}_{\mathcal{D}^{\prime}}$ be the valuation rings in $\mathcal{D}$ and $\mathcal{D}^{\prime}$, respectively, and let $\mathcal{O}_{\mathcal{D}} \rightarrow \Delta$ and $\mathcal{O}_{\mathcal{D}^{\prime}} \rightarrow \Delta^{\prime}$ be the corresponding reduction maps (denoted $x \mapsto \bar{x}$ ). Let $P$ be a maximal subfield of $\Delta$. Since $d$ divides $n$, hence is prime to chark, the extension $P / k$ is separable, and therefore we can find $a \in \mathcal{O}_{\mathcal{D}}$ such that $P=k(\bar{a})$. Set $F=K(a)$. We have

$$
d \geqslant[F: K] \geqslant[P: k]=d,
$$

which implies that $F$ is a maximal subfield of $\mathcal{D}$. By our assumption, $F$ admits a $\mathcal{K}$-embedding into $\mathcal{D}^{\prime}$, and we let $b$ denote the image of $a$ under this embedding. Then the subfield $P^{\prime}=k(\bar{b})$ of $\Delta^{\prime}$ is $k$-isomorphic to $P$ and is maximal as $\operatorname{dim}_{k} \Delta^{\prime}=d^{2}$. Conversely, any maximal subfield of $\Delta^{\prime}$ is $k$-isomorphic to a maximal subfield of $\Delta$. This argument shows that

$$
v_{v}\left(\operatorname{gen}(D) \cap_{n} \operatorname{Br}(K)\right) \subset \operatorname{gen}(\Delta) \cap_{n} \operatorname{Br}(k) .
$$

Since the composition $v_{v} \circ \iota_{k}$ coincides with the identity map on ${ }_{n} \operatorname{Br}(k)$, we conclude that the restriction of $v_{v}$ to $[D]^{-1} \cdot \Lambda \subset \iota_{k}\left({ }_{n} \mathrm{Br}(k)\right)$ is injective. On the other hand, it follows from (5) that

$$
v_{v}\left([D]^{-1} \cdot \Lambda\right) \subset[\Delta]^{-1} \cdot\left(\operatorname{gen}(\Delta) \cap_{n} \operatorname{Br}(k)\right) .
$$

This yields both of the required facts for $\Lambda$ and concludes the proof.

Next, we would like to point out a minor modification of Theorem 3.1, which, under somewhat stronger assumptions, allows one to actually bound the size of $\operatorname{gen}(D)$ and not just that of the intersection $\operatorname{gen}(D) \cap{ }_{n} \operatorname{Br}(K)$. Again, let $D$ be a central division algebra over $K=k(C)$ of dimension $\operatorname{dim}_{K} D=\ell^{2}$, and assume that $[D]$ has exponent $n$ in $\operatorname{Br}(K)$ (of course, $n \mid \ell$, and, moreover, $n$ and $\ell$ have the same prime factors-cf. [11, Proposition 4.5.13]). Clearly $\operatorname{gen}(D) \subset \subset_{\ell} \operatorname{Br}(K)$. Now, if, as before, 
$n$ (and hence $\ell$ ) is prime to char $k$, then the map $\rho_{V}$ on ${ }_{n} \operatorname{Br}(K)$ constructed in the proof of Theorem 2.2 extends to an analogous map $\tilde{\rho}_{V}$ on ${ }_{\ell} \operatorname{Br}(K)$, and we still have the estimate

$$
\left|\tilde{\rho}_{V}(\operatorname{gen}(D))\right| \leqslant \varphi(n)^{r}
$$

Repeating almost verbatim the rest of the proof of Theorem 3.1, we obtain

Theorem 3.2 With notations as above, assume that

- the set $C(k)$ of $k$-rational points is infinite;

- $\left|\ell \operatorname{Br}(K)_{\mathrm{ur}} / \iota_{k}(\ell \operatorname{Br}(k))\right|=: M<\infty$.

Then

(1) if there exists $N<\infty$ such that $|\operatorname{gen}(\Delta)| \leqslant N$ for any central division $k$-algebra $\Delta$ of degree dividing $\ell$, then for any central division $K$-algebra $D$ of degree dividing $\ell$, we have

$$
|\operatorname{gen}(D)| \leqslant M \cdot N \cdot \varphi(n)^{r},
$$

where $r=\operatorname{Ram}_{V}(D)$;

(2) if $\operatorname{gen}(\Delta)$ is finite for any central division $k$-algebra $\Delta$ of degree dividing $\ell$, then $\operatorname{gen}(D)$ is finite for any central division $K$-algebra of degree dividing $\ell$.

One notable case where these results apply is $K=k(x)$, i.e. $C=\mathbb{P}_{k}^{1}$. Strictly speaking, the case of a finite field $k$ is not covered by Theorems 3.1 and 3.2, so let us consider it separately. In this case, the unramified Brauer group $\operatorname{Br}(K)_{V}$ (where, as above, $V$ is the set of all geometric places of $K$ ), is trivial (cf. [11, Corollary 6.4.6] and Sect. 3.6), so we obtain from Theorem 2.2 that for any central division $K$-algebra $D$ of exponent $n$ we have

$$
|\operatorname{gen}(D)| \leqslant \varphi(n)^{r} \text {, where } r=\left|\operatorname{Ram}_{V}(D)\right| \text {. }
$$

Now, let us assume that $k$ is infinite. It is well-known that for any $n$ prime to char $k$, we have ${ }_{n} \operatorname{Br}(K)_{\text {ur }}=\iota_{k}\left({ }_{n} \operatorname{Br}(k)\right)$ (cf. [11, Corollary 6.4.6]), i.e. one can take $M=1$ in Theorems 3.1 and 3.2. We then obtain the following.

Theorem 3.3 Let $K=k(x)$, and let $n>1$ be an integer prime to char $k$.

(1) If there exists $N<\infty$ such that $\left|\operatorname{gen}(\Delta) \cap{ }_{n} \operatorname{Br}(k)\right| \leqslant N$ for any central division $k$-algebra $\Delta$ of exponent $n$, then for any central division $K$-algebra $D$ of exponent $n$, we have

$$
\left|\operatorname{gen}(D) \cap{ }_{n} \operatorname{Br}(K)\right| \leqslant N \cdot \varphi(n)^{r}
$$

where $r=\left|\operatorname{Ram}_{V}(D)\right|$; 
(2) If there exists $N<\infty$ such that $|\operatorname{gen}(\Delta)| \leqslant N$ for any central division $k$-algebra $\Delta$ of degree dividing $n$, then for any central division $K$-algebra $D$ of degree dividing $n$, we have

$$
|\operatorname{gen}(D)| \leqslant N \cdot \varphi(m)^{r},
$$

where $m$ is the exponent of $D$ and $r=\left|\operatorname{Ram}_{V}(D)\right|$;

(3) If $\operatorname{gen}(\Delta) \cap_{n} \operatorname{Br}(k)$ (resp., gen $\left.(\Delta)\right)$ is finite for any central division $k$-algebra $\Delta$ of exponent $n$ (resp., of degree dividing $n$ ), then $\operatorname{gen}(D) \cap{ }_{n} \operatorname{Br}(K)($ resp., $\operatorname{gen}(D)$ ) is finite for any central division $K$-algebra $D$ of exponent $n$ (resp., of degree dividing $n$ ).

Remark 3.4 In [14], Krashen and MacKinnie defined the genus gen' $(D)$ of a central division $K$-algebra $D$ as the collection of $\left[D^{\prime}\right] \in \operatorname{Br}(K)$ having the same finitedimensional splitting fields as $D$ (clearly $\operatorname{gen}^{\prime}(D) \subset \operatorname{gen}(D)$ ). Their Theorem 2.2 provides estimates for $\left|\operatorname{gen}^{\prime}(D)\right|$ of a central division algebra $D$ over $K=k(x)$ of a prime exponent $p \neq$ chark similar to those given in Theorem 3.3.

Of special interest is the question of when $\operatorname{gen}(D)$ reduces to a single element. As we already noted in $\S 1$, this is possible only if $D$ has exponent two and is indeed the case if $D$ is a division algebra of exponent two over a global field (see below). Although over general fields this property may fail even for quaternion algebras [10, $\S 2]$, the following theorem allows one to expand the class of fields over which it does hold.

Theorem 3.5 (Stability Theorem) Let $k$ be a field of characteristic $\neq 2$.

(1) If $k$ satisfies the following property:

(*) If $D$ and $D^{\prime}$ are central division $k$-algebras of exponent 2 having the same maximal subfields then $D \simeq D^{\prime}$ (in other words, for any $D$ of exponent 2, $\left.\left|\operatorname{gen}(D) \cap{ }_{2} \operatorname{Br}(k)\right|=1\right)$.

Then the field of rational functions $k(x)$ also satisfies $(*)$.

(2) If $|\operatorname{gen}(D)|=1$ for any central division $k$-algebra $D$ of exponent 2 , then the same is true for any central division $k(x)$-algebra of exponent 2.

This follows from Theorem 3.3, (1) and (2), with $n=2$ and $N=1$.

\subsection{On the Albert-Brauer-Hasse-Noether theorem}

In this subsection, we will review several consequences of (ABHN) that will be needed in Corollary 3.8 below as well as in the next section. Let $k$ be a global field, and let $V^{k}$ be the set of all places of $k$ (including the archimedean ones if $k$ is a number field). In this case, the set $V^{k} \backslash V_{\infty}^{k}$, where $V_{\infty}^{k}$ is the set of archimedean valuations, satisfies conditions (A) and (B), and the residue map $\rho_{v}$ can be defined on all of $\operatorname{Br}\left(k_{v}\right)$ for any $v \in V^{k} \backslash V_{\infty}^{k}$ because the residue field $\bar{k}_{v}$ is finite, hence perfect. Now, since the absolute Galois group $\mathcal{G}^{(v)}$ of $\bar{k}_{v}$ is isomorphic to $\widehat{\mathbb{Z}}$, one can view $\rho_{v}$ as a map

$$
\rho_{v}: \operatorname{Br}\left(k_{v}\right) \longrightarrow \operatorname{Hom}\left(\mathcal{G}^{(v)}, \mathbb{Q} / \mathbb{Z}\right) \simeq \mathbb{Q} / \mathbb{Z}
$$


This is usually referred to as the invariant map and is well known to be an isomorphism (cf. [27, Chap. 12, §3]). For $v$ archimedean, we have $\operatorname{Br}\left(k_{v}\right)=\mathbb{Z} / 2 \mathbb{Z}$ if $k_{v}=\mathbb{R}$ and $\operatorname{Br}\left(k_{v}\right)=0$ if $k_{v}=\mathbb{C}$, and one defines the invariant map $\rho_{v}$ to be the isomorphism $\mathbb{Z} / 2 \mathbb{Z} \simeq(1 / 2) \mathbb{Z} / \mathbb{Z}$ in the first case and to be the trivial map in the second. Then $(\mathrm{ABHN})$ asserts that the sequence

$$
0 \rightarrow \operatorname{Br}(k) \longrightarrow \bigoplus_{v \in V^{k}} \operatorname{Br}\left(k_{v}\right) \stackrel{\Sigma}{\longrightarrow} \mathbb{Q} / \mathbb{Z} \rightarrow 0
$$

where $\Sigma$ is the sum of the invariant maps, is exact (cf. [3, Chap. 7, 9.6], [19, 18.4], and also [11, 6.5] for the function field case).

It follows from (ABHN) that for a global function field $k$, the unramified Brauer group $\operatorname{Br}(k)_{V}$ with respect to the set $V=V^{k}$ of all (geometric) places of $k$ is trivial. Applying Theorem 2.2, we obtain that $\operatorname{gen}(D)$ is finite for any central division $k$ algebra $D$ and reduces to one element if $D$ is of exponent two ${ }^{1}$ (at least if chark $\neq$ 2 , although the result remains true in characteristic two as well in view of Remark 2.6(2) — we give a direct argument below).

Let now $k$ be a number field. Fix a finite subset $S \subset V^{k}$ containing the set $V_{\infty}^{k}$ of archimedean valuations, and set $V=V^{k} \backslash S$. It follows from (ABHN) that for any $n>1$ we have

$$
{ }_{n} \operatorname{Br}(k)_{V}=\operatorname{Ker}\left(\bigoplus_{v \in S} \operatorname{Br}\left(k_{v}\right) \stackrel{\Sigma_{S}}{\longrightarrow} \frac{1}{n} \mathbb{Z} / \mathbb{Z}\right),
$$

where $\Sigma_{S}$ is the sum of the invariant maps for $v \in S$. Clearly, ${ }_{n} \operatorname{Br}(k)_{V}$ is finite for any $n$ and $S$ as above (and even is a group of exponent g.c.d. $(n, 2)$ for $S=V_{\infty}^{k}$ ), so Theorem 2.2 implies that $\operatorname{gen}(D)$ is finite for any central division $k$-algebra $D$. Unfortunately, ${ }_{2} \operatorname{Br}(k)_{V}$ is nontrivial even for $S=V_{\infty}^{k}$ if $k$ has at least two real places. So, the argument used in the function field case to show that $|\operatorname{gen}(D)|=1$ for any central division $k$-algebra $D$ of exponent two does not apply directly and needs to be modified to take into account the archimedean places. For this, we observe that $(\mathrm{ABHN})$ yields an embedding

$$
0 \rightarrow{ }_{2} \operatorname{Br}(k) \longrightarrow \bigoplus_{v \in V^{k}}{ }_{2} \operatorname{Br}\left(k_{v}\right)
$$

and that ${ }_{2} \operatorname{Br}\left(k_{v}\right)=\mathbb{Z} / 2 \mathbb{Z}$ unless $k_{v}=\mathbb{C}$, in which case $\operatorname{Br}\left(k_{v}\right)=0$. It follows that any $[D] \in{ }_{2} \operatorname{Br}(k)$, represented by a quaternion algebra $D$, is completely determined by the set $\mathcal{R}(D)$ of those $v \in V^{k}$ for which the algebra $D \otimes_{k} k_{v}$ is nontrivial (these are sometimes referred to as the "generalized ramification places"). Furthermore, for $d \in k^{\times} \backslash k^{\times^{2}}$, we have the following well-known criterion:

\footnotetext{
${ }_{1}^{1}$ We note that over a global field $k$, any division algebra $D$ of exponent two is necessarily a quaternion algebra, hence gen $(D) \subset{ }_{2} \operatorname{Br}(k)$.
} 


$$
\ell=k(\sqrt{d}) \text { embeds into } D \Leftrightarrow d \notin k_{v}^{\times^{2}} \text { for all } v \in \mathcal{R}(D) \text {. }
$$

(cf. $[19, \S 18.4$, Corollary b]). Thus, to prove that $|\operatorname{gen}(D)|=1$ for any quaternion division algebra $D$ over $k$, it is enough to show that if two such algebras $D_{1}$ and $D_{2}$ have the same quadratic subfields, then $\mathcal{R}\left(D_{1}\right)=\mathcal{R}\left(D_{2}\right)$. This follows easily from (7) and the weak approximation theorem. Indeed, if, for example, there is a $v_{0} \in \mathcal{R}\left(D_{1}\right) \backslash \mathcal{R}\left(D_{2}\right)$, then using the openness of $k_{v}^{\times 2} \subset k_{v}^{\times}$and weak approximation, one can find $d \in k^{\times} \backslash k^{\times 2}$ such that

$$
d \in k_{v_{0}}^{\times 2} \text { but } d \notin k_{v}^{\times 2} \text { for all } v \in \mathcal{R}\left(D_{2}\right) .
$$

Then according to (7), the quadratic extension $\ell=k(\sqrt{d})$ embeds into $D_{2}$ but not into $D_{1}$, a contradiction.

To make this more concrete, let us consider

Example 3.7 Take the following two quaternion division algebras over $\mathbb{Q}$ :

$$
D_{1}=\left(\frac{-1,3}{\mathbb{Q}}\right) \text { and } D_{2}=\left(\frac{-1,7}{\mathbb{Q}}\right) \text {. }
$$

Then $\mathcal{R}\left(D_{1}\right)=\{2,3\}$ and $\mathcal{R}\left(D_{2}\right)=\{2,7\}$. Clearly, $10 \in \mathbb{Q}_{3}^{\times 2}$ while $10 \notin \mathbb{Q}_{2}^{\times 2}, \mathbb{Q}_{7}^{\times 2}$. So, by (7), the field $\ell=\mathbb{Q}(\sqrt{10})$ embeds into $D_{2}$ but not into $D_{1}$. Thus, $D_{1}$ and $D_{2}$ are distinguished by their quadratic subfields.

Notice that since the subgroup of squares is open in $k_{v}$ for any valuation of any global field of characteristic $\neq 2$, the argument given above works for any such field. To extend it to characteristic two, one needs instead to use the fact that the subgroup $\wp\left(k_{v}\right) \subset k_{v}$ is open, where $\wp(x)=x^{2}-x$, and replace (7) with the observation that for $a \in k \backslash \wp(k)$,

$$
\ell=k\left(\wp^{-1}(a)\right) \text { embeds into } D \Leftrightarrow a \notin \wp\left(k_{v}\right) \text { for all } v \in \mathcal{R}(D) \text {. }
$$

Now, since $|\operatorname{gen}(D)|=1$ for any central division algebra $D$ of exponent two over a global field $k$, Theorem 3.5 yields the following.

Corollary 3.8 Let $k$ be a field of characteristic $\neq 2$ which is either a global field or a finite field, and let $K=k\left(x_{1}, \ldots, x_{r}\right)$ be a finitely generated purely transcendental extension of $k$. Then $|\operatorname{gen}(D)|=1$ for any central division $K$-algebra $D$ of exponent 2.

Remark 3.9. As we already mentioned, Theorem 3 of [5] asserts that if $K$ is a finitely generated field, then for any central division $K$-algebra $D$ of degree $n$ prime to char $K$, the genus $\operatorname{gen}(D)$ is finite. At the same time, generalizing the construction described in $[10, \S 2]$, one can give examples of quaternion division algebras over infinitely generated fields with infinite genus (cf. [18]). So, we would like to point out that Theorems 3.1 and 3.2 can be used to construct examples of division algebras over the function fields of curves with infinitely generated fields of constants having finite 
genus. Some results of this nature are contained in [24, 4.6 and 4.8]. We will not go into details about this here, but would only like to point out that, as follows from the standard exact sequence for the Brauer group of an absolutely irreducible smooth projective curve $C$ over a perfect field $k$ (cf. [16] or [9, (9.25), p. 27]), the requirement $\left|{ }_{n} \operatorname{Br}(K)_{\mathrm{ur}} / \iota_{k}\left({ }_{n} \mathrm{Br}(k)\right)\right|<\infty$ is satisfied, for example, if $k$ is of type (F) as defined by Serre ([26], Chap. 3, Sect. 4.2). Furthermore, the requirement that $C(k)$ is infinite can often be replaced by the much weaker requirement that $\bigcup_{\ell} C(\ell)$, where $\ell$ runs through a family of finite extensions of $k$ of degree prime to $n$, is infinite.

\section{An example: function field of a split elliptic curve}

According to Theorem 2.2, to prove the finiteness of $\operatorname{gen}(D)$ for any central division algebra $D$ of degree $n$ over a field $K$ (provided that $n$ is prime to char $K$ ), it is enough to find a set $V$ of discrete valuations of $K$ that satisfies conditions (A)-(C) and for which the unramified Brauer group ${ }_{n} \operatorname{Br}(K)_{V}$ is finite. As we already mentioned in $\S 1$, in [5] we sketched a proof of the finiteness of ${ }_{n} \operatorname{Br}(K)_{V}$ for a suitable set $V$ of discrete valuations of a given finitely generated field $K$ that relies on results of Deligne and Gabber in étale cohomology. While this proof has the important advantage of giving a lot of flexibility in the choice of $V$, it does not furnish an estimation of the size of ${ }_{n} \operatorname{Br}(K)_{V}$ for any $V$. In this section, we will work out an explicit estimation of $\left|{ }_{2} \operatorname{Br}(K)_{V}\right|$ for a suitable set $V$ of discrete valuations of the field $K$ of rational functions on a split elliptic curve defined over a number field (Theorem 4.1); this yields an estimation of $|\operatorname{gen}(D)|$ for a quaternion algebra $D$ over such $K$ (Corollary 4.11). The method developed in this section can in fact be generalized to arbitrary curves, leading to a more direct proof of the finiteness of ${ }_{n} \operatorname{Br}(K)_{V}$ for a suitable explicitly defined set $V$ of discrete valuations of an arbitrary finitely generated field $K$; details will be published elsewhere. To simplify notations, in this section, given a field $F$, the quaternion algebra $\left(\frac{\alpha, \beta}{F}\right)$ corresponding to a pair $\alpha, \beta$ will be denoted by $(\alpha, \beta)_{F}$.

Let $k$ be a number field, and let $E$ be an elliptic curve over $k$ given by a Weierstrass equation

$$
y^{2}=f(x), \text { where } f(x)=x^{3}+\alpha x^{2}+\beta x+\gamma .
$$

Denote by $\delta \neq 0$ the discriminant of $f$. We will assume in this section that $E$ splits over $k$, i.e. $f$ has three (distinct) roots in $k$. Let

$$
K:=k(E)=k(x, y)
$$

be the function field of $E$. For $s \in k^{\times}$, we let $V^{k}(s)$ denote the finite set $\left\{v \in V^{k} \backslash V_{\infty}^{k} \mid v(s) \neq 0\right\}$. Let us fix a finite set of valuations $S \subset V^{k}$ containing $V_{\infty}^{k} \cup V^{k}(2) \cup V^{k}(\delta)$, as well as all those nonarchimedean $v \in V^{k}$ for which at least one of $\alpha, \beta, \gamma$ has a negative value. For a nonarchimedean $v \in V^{k}$, let $\tilde{v}$ denote its extension to $F:=k(y)$ given by 


$$
\tilde{v}(p(y))=\min _{a_{i} \neq 0} v\left(a_{i}\right) \text { for } p(y)=a_{n} y^{n}+\cdots+a_{0} \in k[y], \quad p \neq 0
$$

(cf. [2, Chap. 6, §10]). Clearly, $K$ is a cubic extension of $F$, and, as we will show in Lemma 4.5 below, for $v \in V^{k} \backslash S$, the valuation $\tilde{v}$ has a unique extension to $K$, which we will denote by $w=w(v)$. We now introduce the following set of discrete valuations of $K$ :

$$
V=V_{0} \cup V_{1} \text {, }
$$

where $V_{0}$ is the set of all geometric places of $K$ (i.e., those discrete valuations that are trivial on $k$ ), and $V_{1}$ consists of the valuations $w(v)$ for all $v \in V^{k} \backslash S$. It is easy to see that $V$ satisfies conditions (A), (B) and (C) of $\S 2$. The main result of this section is the following.

Theorem 4.1 For any finite set $S$ as above, the unramified Brauer group ${ }_{2} \mathrm{Br}(K)_{V}$ is finite of order dividing

$$
2^{|S|-t} \cdot\left|{ }_{2} \mathrm{Cl}_{S}(k)\right|^{2} \cdot\left|U_{S}(k) / U_{S}(k)^{2}\right|^{2},
$$

where $t=c+1$ and $c$ is the number of complex places of $k$, and $\mathrm{Cl}_{S}(k)$ and $U_{S}(k)$ are the class group and the group of units of the ring of $S$-integers $\mathcal{O}_{k}(S)$, respectively.

Our proof will make use of the following description of the geometric Brauer group ${ }_{2} \operatorname{Br}(K)_{V_{0}}$ in the split case, which is valid for any field $k$ of characteristic $\neq 2,3$.

Theorem 4.2 ([4, Theorem 3.6]) Assume that the elliptic curve E given by (8) splits over $k$, i.e.

$$
f(x)=(x-a)(x-b)(x-c) \quad \text { with } a, b, c \in k .
$$

Then

$$
{ }_{2} \operatorname{Br}(K)_{V_{0}}={ }_{2} \operatorname{Br}(k) \oplus I,
$$

where ${ }_{2} \mathrm{Br}(k)$ is identified with a subgroup of ${ }_{2} \mathrm{Br}(K)$ via the canonical map $\operatorname{Br}(k) \rightarrow \operatorname{Br}(K)$, and $I \subset{ }_{2} \operatorname{Br}(K)_{V_{0}}$ is a subgroup such that every element of $I$ is represented by a bi-quaternion algebra of the form

$$
(r, x-b)_{K} \otimes_{K}(s, x-c)_{K}
$$

for some $r, s \in k^{\times}$.

Our argument for Theorem 4.1 will require us to consider separately the ramification properties at places in $V_{1}$ of the constant and bi-quaternionic parts of elements of ${ }_{2} \operatorname{Br}(K)_{V_{0}}$. This analysis will be based on properties of the corestriction map (cf. [25, Chap. 8], [28]). We recall that given a finite separable field extension $K / F$, there is a group homomorphism $\operatorname{cor}_{K / F}: \operatorname{Br}(K) \rightarrow \operatorname{Br}(F)$ with the following properties (cf. [28, Theorems 2.5 and 3.2]): 
(a) the composition

$$
\operatorname{Br}(F) \longrightarrow \operatorname{Br}(K) \stackrel{\operatorname{cor}_{K / F}}{\longrightarrow} \operatorname{Br}(F)
$$

coincides with multiplication by $[K: F]$;

(b) (projection formula) if char $F \neq 2$, then for any $r \in F^{\times}, s \in K^{\times}$, we have

$$
\operatorname{cor}_{K / F}\left(\left[(r, s)_{K}\right]\right)=\left[\left(r, N_{K / F}(s)\right)_{F}\right]
$$

(We note that the projection formula is valid not only for quaternion algebras but for the symbol algebras of any degree $n$ provided that $n$ is prime to char $K$ and $K$ contains a primitive $n$th root of unity).

We will also need the following statement, which easily follows from results proved in [25].

Lemma 4.3 Let $K / F$ be a finite separable field extension, $v$ a discrete valuation of $F$, and $w$ an extension of $v$ to $K$. Assume that

$$
[K: F]=\left[\bar{K}_{w}: \bar{F}_{v}\right]
$$

(then the extension $w$ is automatically unique) and the extension of the residue fields $\bar{K}_{w} / \bar{F}_{v}$ is separable. If $n$ is prime to the characteristic of the residue field $\bar{F}_{v}$ and $[A] \in{ }_{n} \operatorname{Br}(K)$ is unramified at $w$, then $\operatorname{cor}_{K / F}([A])$ is unramified at $v$.

Proof First, (10) implies that the extension $w$ is unique, and therefore the valuation ring $\mathcal{O}_{K, w}$ is the integral closure in $K$ of the valuation ring $\mathcal{O}_{F, v}$. As $K / F$ is separable, it follows that $\mathcal{O}_{K, w}$ is a free $\mathcal{O}_{F, v}$-module of rank $d=[K: F]$. Furthermore, since the extension of residue fields $\bar{K}_{w} / \bar{F}_{v}$ is also separable of degree $d$ (in particular, $w \mid v$ is unramified), by [25, Corollary 2.17], $\mathcal{O}_{K, w}$ is a separable $\mathcal{O}_{F, v}$-algebra. The fact that $[A] \in{ }_{n} \operatorname{Br}(K)$ is unramified at $w$ implies that $A=\mathcal{A} \otimes_{\mathcal{O}_{K, w}} K$ for some Azumaya $\mathcal{O}_{K, w}$-algebra $\mathcal{A}$ (cf. [25, Theorem 10.3]). Then by [25, Theorem 8.1(a)], the corestriction (defined in loc. cit). $\mathcal{B}:=\operatorname{cor}_{\mathcal{O}_{K, w} / \mathcal{O}_{F, v}}(\mathcal{A})$ is an Azumaya $\mathcal{O}_{F, v^{-}}$ algebra, and by $[25$, Theorem $8.1(\mathrm{~d})], \mathcal{B} \otimes_{\mathcal{O}_{F, v}} F$ represents $\operatorname{cor}_{K / F}([A])$, implying that the latter is unramified at $v$ (cf. [25, Theorem 10.3]), as required.

For the rest of the section, we return to the notations $K=k(E)$ and $F=k(y)$. We will need the following two lemmas, the first of which contains a simple computation and the second describes some properties of the valuations $w \in V_{1}$ needed in the proof of Theorem 4.1.

Lemma 4.4 (i) $N_{K / F}(x-a)=N_{K / F}(x-b)=N_{K / F}(x-c)=y^{2}$.

(ii) Let $r \in k^{\times}$and $t \in\{a, b, c\}$, then for the quaternion algebra $(r, x-t)_{K}$ we have

$$
\operatorname{cor}_{K / F}\left(\left[(r, x-t)_{K}\right]\right)=0 \text {. }
$$


Proof (i) We will only prove the claim for $x-a$ as all other cases are treated analogously. It is easy to see that the minimal and characteristic polynomials for $x-a$ over $F$ coincide with

$$
g(t)=t(t+(a-b))(t+(a-c))-y^{2} .
$$

So, $N_{K / F}(x-a)=(-1)^{3} \cdot\left(-y^{2}\right)=y^{2}$.

(ii) Using (i) and the projection formula, we obtain

$$
\operatorname{cor}_{K / F}\left(\left[(r, x-t)_{K}\right]\right)=\left[\left(r, N_{K / F}(x-t)\right)_{F}\right]=\left[\left(r, y^{2}\right)_{F}\right]=0 .
$$

Lemma 4.5 Let $v \in V^{k} \backslash S$, and let $\tilde{v}$ be the extension of $v$ to $F=k(y)$ given by (9).

(i) For any extension $w$ of $\tilde{v}$ to $K$, the residue field extension $\bar{K}_{w} / \bar{F}_{v}$ is a separable cubic extension. Consequently, $\tilde{v}$ has a unique extension (to be denoted $w=$ $w(v))$, which is automatically unramified.

(ii) For $w=w(v)$, the elements $x-a, x-b, x-c$ are units with respect to $w$, and their images $\overline{x-a}, \overline{x-b}$ and $\overline{x-c}$ in $\bar{K}_{w}$ represent distinct nontrivial cosets in $\bar{K}_{w}^{\times} / \bar{K}_{w}^{\times 2}$.

Proof (i) The relation

$$
x^{3}+\alpha x^{2}+\beta x+\left(\gamma-y^{2}\right)=0
$$

implies that $w(x) \geqslant 0$. Reducing, we obtain $\phi(\bar{x})=0$ for the polynomial

$$
\phi(t)=t^{3}+\bar{\alpha} t^{2}+\bar{\beta} t+\left(\bar{\gamma}-\bar{y}^{2}\right)
$$

over $\bar{F}_{\tilde{v}}=\bar{k}_{v}(\bar{y})$, the field of rational functions over the residue field $\bar{k}_{v}$ of $k$. By considering the degree with respect to $\bar{y}$ one finds that $\phi(t)$ has no roots in, hence is irreducible over, $\bar{k}_{v}(\bar{y})$. Furthermore, for the discriminant $\delta(\bar{y})$ of $\phi$ we have $\delta(0)=\bar{\delta}$, where $\delta$ is the discriminant of $f$. So, it follows from our choice of $S$ that $\delta(\bar{y}) \neq 0$, making $\phi$ separable. Thus, $\bar{k}_{v}(\bar{x}, \bar{y})$ is a separable cubic extension of $\bar{k}_{v}(\bar{y})$. By degree considerations, $\bar{K}_{w}=\bar{k}_{v}(\bar{x}, \bar{y})$, and all of our assertions follow.

(ii) Since $w\left(y^{2}\right)=0$ and $w(x) \geqslant 0$,

$$
y^{2}=(x-a)(x-b)(x-c)
$$

yields that $x-a, x-b$ and $x-c$ are all $w$-units. Furthermore, our assumption that $\bar{\delta} \neq 0$ means that the residues $\bar{a}, \bar{b}, \bar{c}$, hence the residues $\bar{x}-\bar{a}, \bar{x}-\bar{b}$ and $\bar{x}-\bar{c}$, are pairwise distinct. First, let us show that no $d \in\{\bar{x}-\bar{a}, \bar{x}-\bar{b}, \bar{x}-\bar{c}\}$ can be a square in $\bar{K}_{w}$. Indeed, if, for example, $d=\bar{x}-\bar{a} \in \bar{K}_{w}^{\times 2}$ then since $d \notin \bar{k}_{v}(\bar{x})^{\times 2}$ and $\left[\bar{K}_{w}: \bar{k}_{v}(\bar{x})\right] \leqslant 2$, we obtain that

$$
\bar{K}_{w}=\bar{k}_{v}(\bar{x})(\bar{y})=\bar{k}_{v}(\bar{x})(\sqrt{d}) .
$$


Consequently,

$$
\frac{\bar{y}^{2}}{d}=(\bar{x}-\bar{b})(\bar{x}-\bar{c}) \in \bar{k}_{v}(\bar{x})^{\times^{2}},
$$

which is impossible as $\bar{b} \neq \bar{c}$. Thus, $\bar{x}-\bar{a}$ is not a square in $\bar{K}_{w}$. Furthermore, if, for example, $\bar{x}-\bar{b}$ and $\bar{x}-\bar{c}$ would represent the same coset modulo $\bar{K}_{w}^{\times 2}$ then $\bar{x}-\bar{a}$ would be a square in $\bar{K}_{w}$, which is not the case.

Remark 4.6 Using the uniqueness statement in [2, Chap. 6, §10, Proposition 2], one easily proves that the restriction of $w$ to $k(x)$ is given by

$$
w(q(x))=\min _{b_{j} \neq 0} v\left(b_{j}\right) \quad \text { for } q(x)=b_{m} x^{m}+\cdots+b_{0} \in k[x] \backslash\{0\} .
$$

We are now in a position to determine when bi-quaternion algebras of the form described in Theorem 4.2 are unramified at places $w \in V_{1}$.

Proposition 4.7 Let $v \in V^{k} \backslash S$, and let $w=w(v)$ be the corresponding valuation of K (see Lemma 4.5). If

$$
\Delta=(r, x-b)_{K} \otimes_{K}(s, x-c)_{K}
$$

is unramified at $w$ then

$$
v(r), v(s) \equiv 0(\bmod 2) .
$$

Proof We will need the following well-known description of the values of the residue map

$$
\rho_{w}:{ }_{2} \operatorname{Br}(K) \longrightarrow \operatorname{Hom}\left(\mathcal{G}^{(w)}, \mathbb{Q} / \mathbb{Z}\right)
$$

(recall that by our construction $\operatorname{char} \bar{K}_{w} \neq 2$ ) on quaternion algebras. For $h \in K^{\times}$ such that $w(h)=0$, we define $\kappa_{h}: \mathcal{G}^{(w)} \rightarrow \mathbb{Z} / 2 \mathbb{Z}$ by

$$
\kappa_{h}(\sigma)=\left\{\begin{array}{ll}
0(\bmod 2) & \text { if } \sigma(\sqrt{\bar{h}})=\sqrt{\bar{h}}, \\
1(\bmod 2) & \text { if } \sigma(\sqrt{\bar{h}})=-\sqrt{\bar{h}},
\end{array} \quad \text { for } \sigma \in \mathcal{G}^{(w)}\right.
$$

where $\bar{h} \in \bar{K}_{w}^{\times}$is the residue of $h$. Then given $g, h \in K^{\times}$with $w(h)=0$, we have

$$
\rho_{w}\left(\left[(g, h)_{K}\right]\right)(\sigma)=\frac{w(g) \kappa_{h}(\sigma)}{2}(\bmod \mathbb{Z}) .
$$

Applying this to $\Delta$ as in the statement of the proposition, we obtain

$$
\rho_{w}([\Delta])(\sigma)=\frac{v(r) \kappa_{(x-b)}(\sigma)+v(s) \kappa_{(x-c)}(\sigma)}{2}(\bmod \mathbb{Z}) .
$$


Since by Lemma 4.5(ii), the elements $\overline{x-b}$ and $\overline{x-c}$ represent different nontrivial cosets in $\bar{K}_{w}^{\times} / \bar{K}_{w}^{\times 2}$, the map

$$
\mathcal{G}^{(w)} \longrightarrow \mathbb{Z} / 2 \mathbb{Z} \times \mathbb{Z} / 2 \mathbb{Z}, \quad \sigma \mapsto\left(\kappa_{(x-b)}(\sigma), \kappa_{(x-a)}(\sigma)\right)
$$

is surjective. Using this in conjunction with the fact that $\rho_{w}([\Delta])$ given by (11) is actually trivial, we easily obtain our claim.

Proof of Theorem 4.1 Let $[D] \in{ }_{2} \operatorname{Br}(K)_{V}$. According to Theorem 4.2, we can write

$$
[D]=\left[\Delta^{\prime} \otimes_{K} \Delta^{\prime \prime}\right]
$$

where $\Delta^{\prime}=\Delta_{0} \otimes_{k} K$ for some central division algebra $\Delta_{0}$ over $k$ such that $\left[\Delta_{0}\right] \in$ ${ }_{2} \operatorname{Br}(k)$, and

$$
\Delta^{\prime \prime}=(r, x-b)_{K} \otimes_{K}(s, x-c)_{K}
$$

for some $r, s \in k^{\times}$. The next lemma describes some restrictions on $\Delta_{0}$ and $\Delta^{\prime \prime}$, which will enable us to limit the number of possibilities for these algebras and eventually prove the theorem.

Lemma 4.8 (i) $\Delta_{0}$ is unramified at all $v \in V^{k} \backslash S$.

(ii) $\Delta^{\prime \prime}$ is unramified at all $w \in V_{1}$.

Proof (i) Fix $v \in V^{k} \backslash S$, and let $w=w(v)$. Since [D] is unramified at $w$, it follows from Lemma 4.3 in conjunction with Lemma 4.5(i) that $\operatorname{cor}_{K / F}([D])$ is unramified at $\tilde{v}$. On the other hand, by Lemma 4.4(ii) we have that $\operatorname{cor}_{K / F}\left(\left[\Delta^{\prime \prime}\right]\right)=0$, and therefore

$$
\operatorname{cor}_{K / F}([D])=\operatorname{cor}_{K / F}\left(\left[\Delta^{\prime}\right]\right)=3 \cdot\left[\Delta_{0} \otimes_{k} F\right]=\left[\Delta_{0} \otimes_{k} F\right]
$$

as $\Delta^{\prime}=\left(\Delta_{0} \otimes_{k} F\right) \otimes_{F} K$. Thus, $\Delta_{0} \otimes_{k} F$ is unramified at $\tilde{v}$, which implies that $\Delta_{0}$ is unramified at $v$, as required. Indeed, since $\left[\Delta_{0}\right] \in{ }_{2} \operatorname{Br}(k)$ and $k$ is a number field, we can take $\Delta_{0}$ to be a quaternion algebra. It follows from the description of the residue map we have already used in the proof of Proposition 4.7 that if $\Delta_{0}$ is ramified at $v$, then it can be written in the form $\Delta_{0}=(r, s)_{k}$, with $r, s \in k^{\times}$, where $v(r)=0$ and $\bar{r} \notin \bar{k}_{v}^{\times^{2}}$ and $v(s)=1$. Then $\Delta_{0} \otimes_{k} F=(r, s)_{F}$. Furthermore, since $\bar{F}_{\tilde{v}}=\bar{k}_{v}(\bar{y})$, we see that $\bar{r} \notin \bar{F}_{\tilde{v}}^{\times^{2}}$. As $\tilde{v}(s)=v(s)=1$, we conclude that $\Delta_{0} \otimes_{k} F$ is ramified at $\tilde{v}$, a contradiction.

(ii) Since $\Delta_{0}$ is unramified at all $v \in V^{K} \backslash S$, it is easy to see (e.g. using Azumaya algebras) that $\Delta^{\prime}$ is unramified at all $w \in V_{1}$. So, $\Delta^{\prime \prime}=(r, x-b)_{K} \otimes_{K}(s, x-c)_{K}$ is unramified at all $w \in V_{1}$.

Thus, the class $\left[\Delta_{0}\right]$ belongs to the unramified Brauer group ${ }_{2} \operatorname{Br}(k)_{V^{k} \backslash S}$. So, the following immediate consequence of $(\mathrm{ABHN})$ bounds the number of possibilities for [ $\left.\Delta_{0}\right]$. 
Lemma 4.9 Let $S \subset V^{k}$ be a finite subset containing $V_{\infty}^{k}$ and at least one non-complex place. Then

$$
\left|{ }_{2} \operatorname{Br}(k)_{V^{k} \backslash S}\right|=2^{|S|-t},
$$

where $t=c+1$ and $c$ is the number of complex places.

Proof According to (6), we have

$$
{ }_{2} \operatorname{Br}(K)_{V^{k} \backslash S} \simeq \operatorname{Ker}\left(\bigoplus_{v \in S}{ }_{2} \operatorname{Br}\left(k_{v}\right) \stackrel{\Sigma_{S}}{\longrightarrow} \frac{1}{2} \mathbb{Z} / \mathbb{Z}\right),
$$

where $\Sigma_{S}$ is the sum of the invariant maps for $v \in S$. Since ${ }_{2} \operatorname{Br}\left(k_{v}\right) \simeq(1 / 2) \mathbb{Z} / \mathbb{Z}$ for every non-complex $v$ and $\Sigma_{S}$ is surjective as $S$ contains a non-complex place, our assertion follows.

To bound the number of possibilities for $\left[\Delta^{\prime \prime}\right]$, we need the following well-known statement.

Lemma 4.10 Let $k$ be a number field and $S \subset V^{k}$ be a finite subset containing $V_{\infty}^{k}$. Set

$$
\tilde{\Gamma}=\left\{x \in k^{\times} \mid v(x) \equiv 0(\bmod 2) \text { for all } v \in V^{k} \backslash S\right\}
$$

If $v_{2}: k^{\times} \rightarrow k^{\times} / k^{\times 2}$ is the canonical homomorphism, then the image $\Gamma=v_{2}(\tilde{\Gamma})$ is finite of order

$$
|\Gamma|=\left|{ }_{2} \mathrm{Cl}_{S}(k)\right| \cdot\left|U_{S}(k) / U_{S}(k)^{2}\right|,
$$

where $\mathrm{Cl}_{S}(k)$ and $U_{S}(k)$ are the class group and the group of units of the ring of $S$-integers $\mathcal{O}_{k}(S)$, respectively.

Proof According to [15, Chap. 6, Theorem 1.4], there is an exact sequence

$$
0 \rightarrow U_{S}(k) / U_{S}(k)^{2} \longrightarrow \Gamma \longrightarrow{ }_{2} \mathrm{Cl}_{S}(k) \rightarrow 0,
$$

from which our claim follows.

By Lemma 4.8(i), [ $\left.\Delta^{\prime \prime}\right]$ is unramified at $w=w(v)$ for any $v \in V^{k} \backslash S$, so it follows from Proposition 4.7 that $r, s \in \tilde{\Gamma}$. Then the number of possibilities for [ $\left.\Delta^{\prime \prime}\right]$ does not exceed $|\Gamma|^{2}$. So, the required estimation in Theorem 4.1 is obtained by combining the estimations from Lemmas 4.9 and 4.10.

Corollary 4.11 In the notations of Theorem 4.1, for any central quaternion division algebra $D$ over $K$, we have

$$
|\operatorname{gen}(D)| \leqslant\left. 2^{|S|-t} \cdot{ }_{2} \mathrm{Cl}_{S}(k)\right|^{2} \cdot\left|U_{S}(k) / U_{S}(k)^{2}\right|^{2} .
$$


Example 4.12 Consider the elliptic curve $E$ over $\mathbb{Q}$ given by $y^{2}=x^{3}-x$. We have $\delta=4$, so one can take $S=\{\infty, 2\}$. Furthermore,

$$
|S|-t=1, \mathrm{Cl}_{S}(\mathbb{Q})=1 \text { and } U_{S}(\mathbb{Q})=\{ \pm 1\} \times \mathbb{Z} .
$$

So, by Corollary 4.11 we have $|\operatorname{gen}(D)| \leqslant 2 \cdot 4^{2}=32$.

\section{Generalizations}

One can generalize the notion of genus from division algebras to algebraic groups using maximal tori in place of maximal subfields. More precisely, given an absolutely almost simple (simply connected or adjoint) algebraic $K$-group $G$, we define its genus as the set of $K$-isomorphism classes of $K$-forms $G^{\prime}$ of $G$ that have the same isomorphism (or isogeny) classes of maximal $K$-tori as $G$ (a different approach to the definition of genus is described in Remark 5.6 below).

Remark 5.1 We note that for $G=\mathrm{SL}_{1, D}$, where $D$ is a finite-dimensional central division $K$-algebra, only maximal separable subfields of $D$ give rise to maximal $K$-tori of $G$. So, to make the definition of $\operatorname{gen}(D)$ consistent with this definition of the genus of $G$, one should probably give the former in terms of maximal separable subfields rather than in terms of all subfields. In this paper, however, we consider only division algebras whose degree is prime to char $K$, for which this issue does not arise, so we opted to use the simplest possible definition of $\operatorname{gen}(D)$.

In view of the finiteness theorem for $\operatorname{gen}(D)$ [5], it seems natural to propose the following.

Conjecture 5.2 Let $G$ be an absolutely almost simple simply connected algebraic group over a finitely generated field $K$ of characteristic zero (or of "good" characteristic relative to $G$ ). Then there exists a finite collection $G_{1}, \ldots, G_{r}$ of $K$-forms of $G$ such that if $H$ is a $K$-form of $G$ having the same isomorphism classes of maximal $K$-tori as $G$, then $H$ is $K$-isomorphic to one of the $G_{i}$ 's.

It was shown in [21, Theorem 7.5] that the conjecture is true if $K$ is a number field. Furthermore, our previous results enable us to prove this conjecture for inner forms of type $A_{\ell}$ in the general case.

Theorem 5.3 Let $G$ be an absolutely almost simple simply connected algebraic group of inner type $A_{\ell}$ over a finitely generated field $K$ whose characteristic is either zero or does not divide $\ell+1$. Then the above conjecture is true for $G$.

Proof We recall that $G=\mathrm{SL}_{m, D}$, where $D$ is a central division $K$-algebra of degree $n$ with $m n=\ell+1$ (cf. [20, 2.3.1]) It is well-known that any maximal $K$-torus $T$ of $G$ is of the form

$$
\mathrm{R}_{E / K}^{(1)}\left(\mathbb{G}_{m}\right)=\mathrm{R}_{E / K}\left(\mathbb{G}_{m}\right) \cap G
$$


(where $\mathbb{G}_{m}$ is the one-dimensional split torus and $\mathrm{R}_{E / K}$ denotes the Weil functor of restriction of scalars) for some maximal étale subalgebra $E$ of $A=M_{m}(D)$. Let $G^{\prime}$ be a $K$-form of $G$ having the same isomorphism classes of maximal $K$-tori as $G$. To prove the theorem, it is enough to establish the following two facts:

(I) $G^{\prime}$ is an inner form over $K$, and consequently $G^{\prime}=\mathrm{SL}_{m, D^{\prime}}$, where $D^{\prime}$ is a central division $K$-algebra of degree $n$;

(II) for any discrete valuation $v$ of $K$, write $D \otimes_{K} K_{v}=M_{S}(\mathcal{D})$ and $D^{\prime} \otimes_{K} K_{v}=$ $M_{s^{\prime}}\left(\mathcal{D}^{\prime}\right)$ with $\mathcal{D}, \mathcal{D}^{\prime}$ division algebras; then $s=s^{\prime}$, and $\mathcal{D}$ and $\mathcal{D}^{\prime}$ have the same isomorphism classes of maximal subfields.

Indeed, according to Theorem 8 in [5], there exists a set $V$ of discrete valuations of $K$ that satisfies conditions (A), (B) and (C) and for which the unramified Brauer group ${ }_{n} \operatorname{Br}(K)_{V}$ is finite. Then as we pointed out in Remark 2.6(1), the local genus $\operatorname{gen}_{V}(D)$ is finite. On the other hand, it follows from (II) that $\left[D^{\prime}\right] \in \operatorname{gen}_{V}(D)$, so the finiteness of the genus of $G$ follows.

Regarding (II), we note that the issue here is that for $T_{i}=\mathrm{R}_{E_{i} / K}^{(1)}\left(\mathbb{G}_{m}\right), i=1,2$ where $E_{1}, E_{2}$ are étale $K$-algebras, a $K$-defined isomorphism $T_{1} \simeq T_{2}$ of tori may not be induced by an isomorphism $E_{1} \simeq E_{2}$ of $K$-algebras. So, the crucial observation is that the former will in fact be (essentially) induced by the latter for generic tori. We will now recall the relevant definitions that apply to an arbitrary semi-simple $K$-group $G$, and then return to the group $G$ as in Theorem 5.3. Let $T$ be a maximal $K$-torus of $G$, and denote by $\Phi=\Phi(G, T)$ the corresponding root system. Furthermore, let $K_{T}$ be the minimal splitting field of $T$ and $\Theta_{T}=\operatorname{Gal}\left(K_{T} / K\right)$ be its Galois group. Then the natural action of $\Theta_{T}$ on the character group $X(T)$ defines a homomorphism

$$
\theta_{T}: \Theta_{T} \longrightarrow \operatorname{Aut}(\Phi)
$$

and we say that $T$ is generic (over $K$ ) if $\operatorname{Im} \theta_{T}$ contains the Weyl group $W(\Phi)=$ $W(G, T)$. If $K$ is a finitely generated field then generic tori always exist; moreover we have the following.

Proposition 5.4 Let $G$ be an absolutely simple algebraic group over a finitely generated field $K$. Given a discrete valuation $v$ of $K$ and a maximal $K_{v}$-torus $T_{v}$ of $G$, there exists a maximal $K$-torus $T$ of $G$ which is generic over $K$ and is conjugate to $T_{v}$ by an element of $G\left(K_{v}\right)$.

If $K$ is of characteristic zero, this is proved in [22, Corollary 3.2]; the argument in positive characteristic requires only minimal changes.

Proof of (I). Let now $G$ be as in Theorem 5.3. Since it is an inner form over $K$, for any maximal $K$-torus $T$ of $G$ we have $\operatorname{Im} \theta_{T} \subset W(G, T)$ (cf. [21, Lemma 4.1b]). Assume now that $G^{\prime}$ is an outer form. Using Proposition 5.4, pick a maximal generic $K$-torus $T^{\prime}$ of $G^{\prime}$. Since $G^{\prime}$ is an outer form, we have $\operatorname{Im} \theta_{T^{\prime}} \not \subset W\left(\Phi\left(G^{\prime}, T^{\prime}\right)\right)$, and therefore eventually $\operatorname{Im} \theta_{T^{\prime}}=\operatorname{Aut}\left(\Phi\left(G^{\prime}, T^{\prime}\right)\right)$. It follows from our previous remark that $T^{\prime}$ cannot be $K$-isomorphic to any maximal $K$-defined torus of $G$, a contradiction. Thus, $G^{\prime}=\mathrm{SL}_{m^{\prime}, D^{\prime}}$. Furthermore, the fact that $G$ and $G^{\prime}$ have the same isomorphism classes of maximal $K$-tori, implies that

$$
m-1=\operatorname{rk}_{K} G=\operatorname{rk}_{K} G^{\prime}=m^{\prime}-1,
$$


i.e. $m=m^{\prime}$, and hence $D^{\prime}$ has degree $n$.

Proof of (II). Let $A=M_{m}(D)$ and $A^{\prime}=M_{m}\left(D^{\prime}\right)$. It is enough to show that for a discrete valuation $v$ of $K$, the algebras $\mathcal{A}_{v}=A \otimes_{K} K_{v}$ and $\mathcal{A}_{v}^{\prime}=A^{\prime} \otimes_{K} K_{v}$ have the same isomorphism classes of maximal étale subalgebras (cf. the Lemma 2.3 and Corollary 2.4 in [24]). Fix a maximal étale $K_{v}$-subalgebra $\mathcal{E}$ of $\mathcal{A}_{v}$, and let $\mathcal{T}=\mathrm{R}_{\mathcal{E} / K_{v}}^{(1)}\left(\mathbb{G}_{m}\right)$ be the corresponding maximal $K_{v}$-torus of $G$. Using Proposition 5.4, we can find a maximal $K$-torus $T$ of $G$ which is generic over $K$ and which is conjugate to $\mathcal{T}$ by an element of $G\left(K_{v}\right)$. We have $T=\mathrm{R}_{E / K}^{(1)}\left(\mathbb{G}_{m}\right)$ for some maximal étale $K$-subalgebra $E$ of $A$, and then $E \otimes_{K} K_{v}$ and $\mathcal{E}$ are isomorphic as $K_{v}$-algebras. By our assumption, there exists a $K$-isomorphism $\varphi: T \rightarrow T^{\prime}$ onto a maximal $K$ torus $T^{\prime}$ of $G^{\prime}$, where $T^{\prime}=\mathrm{R}_{E^{\prime} / K}\left(\mathbb{G}_{m}\right)$. Since $T$ is generic and $G, G^{\prime}$ are inner forms, it follows from Lemma 4.3 and Remark 4.4 in [21] that $\varphi$ extends to an isomorphism $\tilde{\varphi}: G \rightarrow G^{\prime}$ defined over an algebraic closure $\bar{K}$ of $K$. Now, pick an isomorphism $A \otimes_{K} \bar{K} \rightarrow A^{\prime} \otimes_{K} \bar{K}$ of $\bar{K}$-algebras, and let $\varphi_{0}: G \rightarrow G^{\prime}$ be the corresponding $\bar{K}$-isomorphism of algebraic groups. Set $\varphi^{\prime}=\varphi$ if $\psi=\varphi_{0}^{-1} \circ \tilde{\varphi} \in$ Aut $G$ is inner, and define $\varphi^{\prime}$ by $\varphi^{\prime}(t)=\varphi(t)^{-1}$ for $t \in T$ if $\psi$ is outer. Then in either case, $\varphi^{\prime}: T \rightarrow T^{\prime}$ is a $K$-defined isomorphism of tori that extends to a $\bar{K}$-isomorphism $\tilde{\varphi}^{\prime}: G \rightarrow G^{\prime}$ induced by some isomorphism of $\bar{K}$-algebras $\tau: A \otimes_{K} \bar{K} \rightarrow A^{\prime} \otimes_{K} \bar{K}$. Since $E$ (resp., $E^{\prime}$ ) coincides with the $K$-subalgebra of $A$ (resp., $A^{\prime}$ ) generated by $T(K)$ (resp., $T^{\prime}(K)$ ), and $\varphi^{\prime}(T(K))=T^{\prime}(K)$, we conclude that $\tau$ yields a $K$-isomorphism between $E$ and $E^{\prime}$. It follows that $\mathcal{E}$ is isomorphic to the maximal étale $K_{v}$-subalgebra $E^{\prime} \otimes_{K} K_{v}$ of $\mathcal{A}_{v}^{\prime}$. By symmetry, we see that that $\mathcal{A}_{v}$ and $\mathcal{A}_{v}^{\prime}$ have the same isomorphism classes of maximal étale $K_{v}$-subalgebras, as required.

Remark 5.5 In the notations introduced in the proof of (II) above, it follows from Lemma 4.3 and Remark 4.4 in [21] that if there exists a nontrivial $K$-defined isogeny $T \rightarrow T^{\prime}$ of generic tori in absolutely almost simple simply connected groups of type $\mathrm{A}_{\ell}$ then there also exists a $K$-defined isomorphism $T \rightarrow T^{\prime}$. So, the finiteness result of Theorem 5.3 remains valid if one defines the genus using isogeny classes in place of isomorphism classes. In fact, it also remains valid if one defines the genus in terms of isomorphism/isogeny classes of just maximal generic tori. On the other hand, the argument used to prove Theorem 1 in [21] shows that if $G$ and $G^{\prime}$ are absolutely almost simple algebraic groups over a finitely generated field $K$ with the same isogeny classes of maximal $K$-tori then either they have the same type, or one of them is of type $\mathrm{B}_{\ell}$ and the other of type $\mathrm{C}_{\ell}$ for some $\ell \geqslant 3$. In particular, if $G$ is an absolutely almost simple simply connected algebraic group of type different from $\mathrm{B}_{\ell}$ and $\mathrm{C}_{\ell}(\ell \geqslant 3)$ over a finitely generated field $K$, then any absolutely almost simple simply connected $K$-group having the same isogeny classes of maximal $K$-tori as $G$ is necessarily a $K$-form of $G$.

Remark 5.6 (Due to A.S. Merkurjev) One can offer a different (in a way, more functorial) definition of the genus of an absolutely almost simple $K$-group $G$ as the set of $K$-isomorphism classes of $K$-forms $G^{\prime}$ of $G$ that have the same isomorphism/isogeny classes of maximal tori not only over $K$ but also over any field extension $F / K$. The well-known theorem of Amitsur [1] asserts that if $D$ and $D^{\prime}$ are finite-dimensional central division $K$-algebras such that every field extension $F / K$ which splits $D$ also 
splits $D^{\prime}$ then $\left[D^{\prime}\right]$ lies in the cyclic subgroup $\langle[D]\rangle$ of $\operatorname{Br}(K)$ generated by $[D]$; in particular, the genus of $G=\mathrm{SL}_{1, D}$ would then be finite for any $D$ and would reduce to one element for $D$ of exponent two. Furthermore, according to a result of Izhboldin [12], given nondegenerate quadratic forms $q$ and $q^{\prime}$ of odd dimension $n$ over a field $K$ of characteristic $\neq 2$ the following condition

( $q$ and $q^{\prime}$ have the same Witt index over any extension $F / K$,

implies that $q$ and $q^{\prime}$ are scalar multiples of each other (this conclusion being false for even-dimensional forms). It follows that for $G=\operatorname{Spin}_{n}(q)$ with $n$ odd the genus of $G$ as defined in this remark reduces to a single element. We note that the condition $(\star)$ is equivalent to the fact that the motives of $q$ and $q^{\prime}$ in the category of Chow motives are isomorphic (Vishik [31], and also Vishik [32, Theorem 4.18], Karpenko [13]), so one can call the genus defined above the motivic genus. It would be interesting to investigate the motivic genus for other types of algebraic groups; e.g. one can expect it trivial for type $\mathrm{C}_{\ell}$ (note that it easily follows from properties of the Pfister forms that it is always trivial for type $\mathrm{G}_{2}$, at least in characteristic not 2).

\section{A geometric connection}

The recent interest in the problem of determining an absolutely almost simple algebraic $K$-group by the isomorphism/isogeny classes of its maximal $K$-tori (in particular, of determining a finite-dimensional central division $K$-algebra by the isomorphism classes of maximal subfields) was motivated at least in part by the investigation of length-commensurable and isospectral locally symmetric spaces (cf. [23, §6]). In fact, the initial question of whether two central quaternion division algebras over $\mathbb{Q}(x)$ with the same quadratic subfields are necessarily isomorphic appeared in print (apparently, for the first time) in the paper [21] on locally symmetric space (although unofficially it may have been around for some time). The purpose of this section is to briefly recall some aspects of this geometric connection in order to provide a context for a general conjecture about Zariski-dense subgroups.

For a (compact) Riemannian manifold $M$, we let $\mathcal{E}(M)$ denote the Laplace spectrum of $M$ (i.e, the spectrum of the Beltrami-Laplace operator) and $L(M)$ the (weak) length spectrum of $M$ (i.e., the collection of lengths of all closed geodesics in $M$ ). It is a classical problem in differential geometry to determine what one can say about two Riemannian manifolds $M_{1}$ and $M_{2}$ given the fact that they are isospectral, i.e. $\mathcal{E}\left(M_{1}\right)=\mathcal{E}\left(M_{2}\right)$. It turns out that for locally symmetric spaces, isospectrality implies iso-length-spectrality $\left(L\left(M_{1}\right)=L\left(M_{2}\right)\right)$, see [21, Theorem 10.1]. On the other hand, neither iso-length-spectrality nor even isopectrality typically implies that $M_{1}$ and $M_{2}$ are isometric, although it was established in [21] that for arithmetically defined locally symmetric spaces of simple real algebraic groups of types different from $A_{\ell}, D_{2 \ell+1}$ $(\ell>1)$ and $\mathrm{E}_{6}$, the weaker condition of length-commensurability $\left(\mathbb{Q} \cdot L\left(M_{1}\right)=\right.$ $\left.\mathbb{Q} \cdot L\left(M_{2}\right)\right)$ already implies that $M_{1}$ and $M_{2}$ are commensurable, i.e. have a common finite-sheeted cover. We refer the reader to [23] for the history of the problem and an exposition of the available results. We note only that the problem remains wide open for non-arithmetically defined locally symmetric spaces, and will now show that 
its analysis in the case of Riemann surfaces leads to a variant of the problem about quaternion algebras with the same maximal fields.

Let $\mathbb{H}=\{x+i y \in \mathbb{C} \mid y>0\}$ be the upper half-plane with the standard hyperbolic metric

$$
d s^{2}=y^{-2}\left(d x^{2}+d y^{2}\right)
$$

Any compact Riemann surface $M$ of genus $g>1$ can be written as a quotient $M=\mathbb{H} / \Gamma$ by a (cocompact) discrete torsion-free subgroup $\Gamma \subset P S L_{2}(\mathbb{R})$. Let $\pi: S L_{2}(\mathbb{R}) \rightarrow P S L_{2}(\mathbb{R})$ be the canonical homomorphism, and set $\tilde{\Gamma}=\pi^{-1}(\Gamma)$. It is well-known that closed geodesics in $M$ correspond to nontrivial semi-simple elements in $\Gamma$; the precise nature of this correspondence is not important for us, as we only need information about the length. One shows that if $c_{\gamma}$ is a closed geodesic in $M$ corresponding to a nontrivial semi-simple element $\gamma \in \Gamma$, then its length is given by the formula:

$$
\ell\left(c_{\gamma}\right)=\frac{2}{n_{\gamma}} \cdot|\log | t_{\tilde{\gamma}}||
$$

where $n_{\gamma}$ is an integer $\geqslant 1$ (winding number) and $t_{\tilde{\gamma}}$ is an eigenvalue of an element $\tilde{\gamma} \in \tilde{\Gamma}$ such that $\pi(\tilde{\gamma})=\gamma$ (note that since $\Gamma$ is torsion-free and discrete, $\tilde{\gamma}$ is automatically hyperbolic, i.e. $t_{\tilde{\gamma}} \in \mathbb{R}$ and $\tilde{\gamma}$ is conjugate in $S L_{2}(\mathbb{R})$ to $\left(\begin{array}{cc}t_{\tilde{\gamma}} & 0 \\ 0 & t_{\tilde{\gamma}}^{-1}\end{array}\right)$ ). Thus,

$$
\mathbb{Q} \cdot L(M)=\mathbb{Q} \cdot\left\{\log \left|t_{\tilde{\gamma}}\right| \mid \gamma \in \Gamma \backslash\{1\} \text { semi-simple }\right\} .
$$

One of the tools for analyzing Kleinian groups developed in [17] is based on associating to a Zariski-dense subgroup $\tilde{\Gamma} \subset S L_{2}(\mathbb{R})\left(\right.$ or $\left.S L_{2}(\mathbb{C})\right)$ the $\mathbb{Q}$-subalgebra $D=\mathbb{Q}\left[\tilde{\Gamma}^{(2)}\right]$ of $M_{2}(\mathbb{R})$ (or $M_{2}(\mathbb{C})$ ) spanned by the subgroup $\tilde{\Gamma}^{(2)} \subset \tilde{\Gamma}$ generated by squares. This algebra turns out to be a quaternion algebra whose center is the trace field $K=K_{\tilde{\Gamma}^{(2)}}$ (the subfield generated over $\mathbb{Q}$ by the traces $\operatorname{tr} \tilde{\gamma}$ for $\tilde{\gamma} \in \tilde{\Gamma}^{(2)}$ ), cf. [17, 3.1, 3.2]; note that $K$ is automatically contained in $D$ as for any $\tilde{\gamma} \in \mathrm{SL}_{2}$ we have

$$
\tilde{\gamma}+\tilde{\gamma}^{-1}=(\operatorname{tr} \tilde{\gamma}) \cdot I_{2}
$$

(The reason for passing from $\tilde{\Gamma}$ to $\tilde{\Gamma}^{(2)}$ can be seen in the fact that the "true" field of definition of a Zariski-dense subgroup is generated by the traces in the adjoint representation, cf. [29]). If the subgroup $\Gamma$ is arithmetic then $D$ is precisely the quaternion algebra involved in its description, so one can expect $D$ to play a significant role also in the general case. Finally, we note that for any semisimple $\tilde{\gamma} \in \tilde{\Gamma}^{(2)} \backslash\{ \pm 1\}$, the subalgebra $K[\tilde{\gamma}]$ is a maximal étale subalgebra of $D$ and that for any integer $n \neq 0$ we have $K\left[\tilde{\gamma}^{n}\right]=K[\tilde{\gamma}]$.

Now, let $M_{i}=\mathbb{H} / \Gamma_{i}(i=1,2)$ be two compact Riemann surfaces as above, and let $D_{i}=\mathbb{Q}\left[\tilde{\Gamma}_{i}^{(2)}\right]$ and $K_{i}=Z\left(D_{i}\right)$, for $i=1,2$, be the corresponding quaternion algebra 
and trace field, respectively. Assume that $M_{1}$ and $M_{2}$ are length-commensurable. Then it follows, for example, from [21, Theorem 2] that

$$
K_{1}=K_{2}=: K
$$

Furthermore, (13) implies that for any $\tilde{\gamma}_{1} \in \tilde{\Gamma}_{1}^{(2)}$, there exists $\tilde{\gamma}_{2} \in \tilde{\Gamma}^{(2)}$ such that

$$
t_{\tilde{\gamma}_{1}}^{m}=t_{\tilde{\gamma}_{2}}^{n}
$$

for some nonzero integers $m, n$, and vice versa. Then the elements $\gamma_{1}^{m}$ and $\gamma_{2}^{n}$ are conjugate in $S L_{2}(\mathbb{R})$, hence

$$
K\left[\tilde{\gamma}_{1}\right]=K\left[\tilde{\gamma}_{1}^{m}\right] \simeq K\left[\tilde{\gamma}_{2}^{n}\right]=K\left[\tilde{\gamma}_{2}\right]
$$

Thus, the length-commensurability of $M_{1}$ and $M_{2}$ translates into the following condition: $D_{1}$ and $D_{2}$ have the same isomorphism classes of maximal étale subalgebras that intersect nontrivially ${ }^{2} \tilde{\Gamma}_{1}^{(2)}$ and $\tilde{\Gamma}_{2}^{(2)}$, respectively. On the other hand, if $M_{1}$ and $M_{2}$ are commensurable, then $D_{1}$ and $D_{2}$ are isomorphic as $K$-algebras (cf. [17]). So, the expected (but currently lacking much supporting evidence) result that the compact Riemann surfaces that are length-commensurable to a given compact Riemann surface form finitely many commensurability classes leads to the following algebraic problem:

(*) Let $D$ be a central quaternion algebra over a finitely generated field $K$, and $\Gamma \subset$ $S L(1, D)$ be a finitely generated subgroup Zariski-dense in $G=\mathrm{SL}_{1, D}$ with the trace field $K$. Let gen $(D, \Gamma)$ denote the collection of classes $\left[D^{\prime}\right] \in \operatorname{Br}(K)$ where $D^{\prime}$ is a quaternion $K$-algebra for which there exists a finitely generated subgroup $\Gamma^{\prime} \subset S L\left(1, D^{\prime}\right)$ Zariski-dense in $G^{\prime}=\mathrm{SL}_{1, D^{\prime}}$ with the trace field $K$ such that $D$ and $D^{\prime}$ have the same isomorphism classes of étale subalgebras that nontrivially intersect $\Gamma$ and $\Gamma^{\prime}$, respectively. Then $\operatorname{gen}(D, \Gamma)$ is finite.

(Of course, the commensurability class of a nonarithmetic $\Gamma$ is not determined uniquely by the corresponding quaternion algebra $D$; in fact, E.B. Vinberg [30] has constructed an infinite family of pairwise noncommensurable cocompact lattices in $S L_{2}(\mathbb{R})$ that are contained in $M_{2}(\mathbb{Q})$. On the other hand, one can consider the following stronger "asymmetric" version of (*): Let $D, K$ and $\Gamma$ be as in $(*)$. Define $\operatorname{gen}^{\prime}(D, \Gamma)$ to be the collection of classes $\left[D^{\prime}\right] \in \operatorname{Br}(K)$ where $D^{\prime}$ is a central quaternion division $K$-algebra with the following property: any maximal subfield $P$ of $D$ that is generated by an element of $\Gamma$ admits a $K$-embedding into $D^{\prime}$. Then $\operatorname{gen}^{\prime}(D, \Gamma)$ is finite).

We will now indicate how $(*)$ can be generalized to arbitrary absolutely almost simple groups. For this, the relation between the elements $\tilde{\gamma}_{1}$ and $\tilde{\gamma}_{2}$ expressed by (14) needs to be replaced by the notion of weak commensurability introduced in [21]. Now, rather than working directly with the definition given there, it will be

\footnotetext{
$\overline{2 \text { I.e. contain a common element }} \neq \pm 1$.
} 
more convenient to start with an equivalent form of this notion that was used in $[23,2.2]$. Let $G_{1} \subset \mathrm{GL}_{N_{1}}$ and $G_{2} \subset \mathrm{GL}_{N_{2}}$ be two semi-simple algebraic groups defined over a field $F$ of characteristic zero. Semi-simple elements $\gamma_{1} \in G_{1}(F)$ and $\gamma_{2} \in G_{2}(F)$ are said to be weakly commensurable if the subgroups of $\bar{F}^{\times}$generated by their eigenvalues intersect nontrivially. Obviously, this notion is a direct generalization of the condition (14); at the same time, it is equivalent to the more technical definition given in $[21, \S 1]$ that requires the existence of maximal $F$-tori $T_{i}$ of $G_{i}$, for $i=1,2$, such that $\gamma_{i} \in T_{i}(F)$ and for some characters $\chi_{i} \in X\left(T_{i}\right)$, we have

$$
\chi_{1}\left(\gamma_{1}\right)=\chi_{2}\left(\gamma_{2}\right) \neq 1
$$

Furthermore, (Zariski-dense) subgroups $\Gamma_{1} \subset G_{1}(F)$ and $\Gamma_{2} \subset G_{2}(F)$ are weakly commensurable if every semi-simple element $\gamma_{1} \in \Gamma_{1}$ of infinite order is weakly commensurable to some semi-simple element $\gamma_{2} \in \Gamma_{2}$ of infinite order, and vice versa. We refer the reader to [21] and the survey article [23] for results about weakly commensurable Zariski-dense subgroups, one of which (see [21, Theorem 2]) states that weakly commensurable finitely generated Zariski-dense subgroups of absolutely almost simple algebraic groups have the same trace field (defined in terms of the adjoint representation); we only mention here that, just as in the case of Riemann surfaces, length-commensurability of locally symmetric spaces of simple real algebraic groups is adequately reflected by weak commensurability of their fundamental groups (see $[23,2.3]$ for a discussion). Now, we would like to propose the following conjecture generalizing $(*)$.

Conjecture 6.1 Let $G_{1}$ and $G_{2}$ be absolutely simple (hence adjoint) algebraic groups over a field $F$ of characteristic zero, let $\Gamma_{1} \subset G_{1}(F)$ be a finitely generated Zariskidense subgroup, and let $K=K_{\Gamma_{1}}$ be the trace field ${ }^{3}$ of $\Gamma_{1}$. Then there exists a finite collection $\mathcal{G}_{2}^{(1)}, \ldots, \mathcal{G}_{2}^{(r)}$ of $F / K$-forms of $G_{2}$ such that if $\Gamma_{2} \subset G_{2}(F)$ is a finitely generated Zariski-dense subgroup that is weakly commensurable to $\Gamma_{1}$, then it is conjugate to a subgroup of one of the $\mathcal{G}_{2}^{(i)}(K)^{\prime} s\left(\subset G_{2}(F)\right)$.

The connection between the analysis of weak commensurability and the study of absolutely almost simple algebraic groups having the same isomorphism/isogeny classes of maximal tori (and hence between Conjectures 5.2 and 6.1) is discussed in [23, §9]. We note that Weisfeiler's Approximation Theorem [35], in conjunction with finiteness results for Galois cohomology of algebraic groups over number fields (cf. $[21, \S 6])$, allows one to prove Conjecture 6.1 for $K$ a number field-details will be published elsewhere. No other cases have been considered so far, but we hope that the techniques involved in the proof of Theorem 5.3 will lead to a proof of Conjecture 6.1 for inner forms of type $A_{\ell}$ (of course, one can also consider stronger asymmetric versions of Conjectures 5.2 and 6.1 along the lines indicated after the statement of $(*))$.

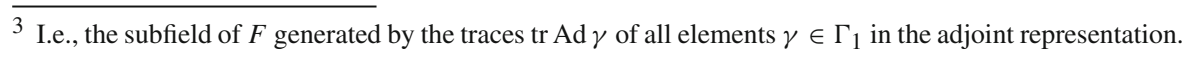


Acknowledgments We are grateful to A.S. Merkurjev for useful discussions. The first-named author was partially supported by the Canada Research Chairs program and by an NSERC research grant. The secondnamed author was partially supported by NSF grant DMS-0965758, BSF grant 2010149 and the Humboldt Foundation. During the preparation of the final version of this paper he was visiting the Mathematics Department at Yale whose hospitality and support are thankfully acknowledged.

Open Access This article is distributed under the terms of the Creative Commons Attribution License which permits any use, distribution, and reproduction in any medium, provided the original author(s) and the source are credited.

\section{References}

1. Amitsur, S.: Generic splitting fields of central simple algebras. Ann. math. 62, 8-43 (1955)

2. Bourbaki, N.: Commutative algebra, ch. 1-7. Springer, New York (1989)

3. Cassels, J.W.S., Fröhlich, A. (eds.): Algebraic number theory. Lond. Math. Soc. (2010)

4. Chernousov, V., Guletskii, V.: 2-Torsion of the Brauer group of an elliptic curve. Doc. Math. (2001) (extra volume, 85-120)

5. Chernousov, V.I., Rapinchuk, A.S., Rapinchuk, I.A.: On the genus of a division algebra. C.R. Acad. Sci. Paris Ser. I 350, 807-812 (2012)

6. Colliot-Thélène, J.-L.: Private communication (2012)

7. Deligne, P.: Cohomologie étale, SGA $4 \frac{1}{2}$. Lect. Notes Math. 569 (1977) (Springer)

8. Fujiwara, K.: A proof of the absolute purity conjecture (after Gabber), Algebraic Geometry 2000, Azumino (Hotaka). Adv. Stud. Pure Math. 36, 153-183 (2002) (Math. Soc. Jpn)

9. Garibaldi, S., Merkurjev, A., Serre, J.-P.: Cohomological invariants in Galois cohomology, University lecture series, 28, AMS (2003)

10. Garibaldi, S., Saltman, D.: Quaternion algebras with the same subfields, quadratic forms, linear algebraic groups, and cohomology. Dev. Math. 18, 225-238 (2010) (Springer, New York)

11. Gille, P., Szamuely, T.: Central simple algebras and Galois cohomology. Cambridge University, Cambridge (2006)

12. Izhboldin, O.T.: Motivic equivalence of quadratic forms. Doc. Math. 3, 341-351 (1998)

13. Karpenko, N.: Criteria of motivic equivalence for quadratic forms and central simple agebras. Math. Ann. 317(3), 585-611 (2000)

14. Krashen, D., McKinnie, K.: Distinguishing division algebras by finite splitting fields. Manuscr. Math. 134, 171-182 (2011)

15. Lang, S.: Fundamentals of diophantine geometry. Springer, New York (1983)

16. Lichtenbaum, S.: Duality theorems for curves over $p$-adic fields. Invent. Math. 7, 120-136 (1969)

17. Maclachlan, C., Reid, A.W.: The arithmetic of hyperbolic 3-manifolds. GTM 219 (2003) (Springer)

18. Meyer, J.S.: A division algebra with infinite genus (arXiv:1301.5632)

19. Pierce, R.S.: Associative algebras. GTM 88 (1982) (Springer)

20. Platonov, V.P., Rapinchuk, A.S.: Algebraic groups and number theory. Academic Press, San Diego (1994)

21. Prasad, G., Rapinchuk, A.S.: Weakly commensurable arithmetic groups and isospectral locally symmetric spaces. Publ. Math. IHES 109, 113-184 (2009)

22. Prasad, G., Rapinchuk, A.S.: On the fields generated by the lengths of closed geodesics in locally symmetric spaces (arXiv:1110.0141)

23. Prasad, G., Rapinchuk, A.S.: Generic elements in Zariski-dense subgroups and isospectral locally symmetric spaces (arXiv:1212.1217)

24. Rapinchuk, A.S., Rapinchuk, I.A.: On division algebras having the same maximal subfields. Manuscr. Math. 132, 273-293 (2010)

25. Saltman, D.: Lectures on division algebras, CBMS regional conference series. 94 (1999) (AMS)

26. Serre, J.-P.: Galois cohomology. Springer, New York (1997)

27. Serre, J.-P.: Local fields. GTM 67 (1979) (Springer)

28. Tignol, J.-P.: On the corestriction of central simple algebras. Math. Z. 194, 267-274 (1987)

29. Vinberg, E.B.: Rings of definition of dense subgroups of semisimple linear groups. Math. USSR Izv. 5, 45-55 (1971) 
30. Vinberg, E.B.: Some examples of Fuchsian groups sitting in $S L_{2}(\mathbb{Q})$, preprint 2011 of the SFB-701. Universität Bielefeld (2012)

31. Vishik, A.: Integral motives of quadrics. Max Planck Institute für Mathematik (preprint MPI-1998-13, pp. 1-82), Bonn (1998)

32. Vishik, A.: Motives of quadrics with applications to the theory of quadratic forms, Geometric methods in the algebraic theory of quadratic forms, LNM 1835, 25-101, Springer (2004)

33. Wadsworth, A.R.: Extending valuations to finite-dimensional division algebras. Proc. AMS 98(1), 20-22 (1986)

34. Wadsworth, A.R.: Valuation theory on finite dimensional division algebras. In: Saskatoon, S.K. (ed.) Valuation theory and its applications, vol. 1. (1999). Fields Inst. Commun. 32, 385-449 (Am. Math. Soc. Provid. RI) (2002)

35. Weisfeiler, B.: Strong approximation for Zariski-dense subgroups of semi-simple algebraic groups. Ann. math. 120(2), 271-315 (1984) 